\title{
Schwarz Reflection Geometry II: Local and Global Behavior of the Exponential Map
}

\author{
Annalisa Calini and Joel Langer
}

\section{CONTENTS}

1. Introduction

2. Analytic Jordan Curves and Their Schwarz Functions

3. Geodesics in $\Lambda$

4. Holomorphic Flows and Trajectories of Differentials

5. Maximal Geodesics and Singular Ring Domains

6. The Infinitesimal Symmetric Space $T_{\circ} \Lambda$

7. Geodesics Generated by Rational Vector Fields

8. Analytic Diffeomorphisms of $S^{\mathbf{1}}$

9. Real Meromorphic Differentials on $S^{\mathbf{1}}$

10. Equivalence of Real Meromorphic Differentials on $S^{\mathbf{1}}$ References
2000 AMS Subject Classification: 53C35, 53A30, 30D05

Keywords: Schwarz reflection, symmetric space, Witt algebra
A local normal form is obtained for geodesics in the space $\Lambda=\{\Gamma\}$ of analytic Jordan curves in the extended complex plane with symmetric space multiplication $\Gamma_{1} \cdot \Gamma_{2}$ defined by Schwarzian reflection of $\Gamma_{2}$ in $\Gamma_{1}$. Local geometric features of $(\Lambda, \cdot)$ will be seen to reflect primarily the structure of the Witt algebra, while issues of global behavior of the exponential map will be viewed in the context of conformal mapping theory.

\section{INTRODUCTION}

Geometry springs from many sources: symmetry defines the homogeneous geometries of Möbius, Lie, and Klein; measurement is the essence of the inhomogeneous spaces of Riemann and Einstein; connections are basic to the more general geometries of Cartan and to twentiethcentury developments such as gauge theory.

A more specialized source of geometric structure, reflection, generates a subclass of the former homogeneous geometries; namely, a symmetric space may be defined simply as a manifold with multiplication $p \cdot q=$ reflection of $q$ in $p$ (such that for any point $p$, left multiplication $s_{p}=p$. is an involutive automorphism with isolated fixed point $p$ ). As developed in [Loos 69], all elements of symmetric space geometry - the homogeneous and (when present) metric structures, the connection, curvature, geodesics, etc.- then follow as consequences of such multiplication.

The present work is the second part of an ongoing exploration of Schwarzian reflection as a source of geometry on an infinite-dimensional space $\Lambda$ comprising analytic curves in the complex plane. The development is not straightforward because many standard symmetricspace constructions are generally valid only in the finitedimensional setting. For instance, heuristics tempt one to view the space of unparametrized, analytic, closed curves as a quotient space $\Lambda=G / H$-analytic embeddings of the unit circle $S^{1}$ into $\mathbb{C}$ modulo analytic diffeomorphisms

(C) A K Peters, Ltd. 1058-6458/2007 \$0.50 per page Experimental Mathematics 16:3, page 321 
of $S^{1}$ (reparametrizations) - but unfortunately, the analytic embeddings do not form a group (put another way, the only candidate for $G$ would be the complexification $H_{\mathbb{C}}$ of the diffeomorphism group $H$, which does not exist).

Nevertheless, as shown in [Calini and Langer 03], many elements of symmetric space theory apply nicely to $\Lambda$ on a formal level and yield locally meaningful equations. Thus, one of our underlying goals is to see how far "standard" geometric interpretations of solutions to such equations may be pushed, as well as to explore "nonstandard" interpretations for untoward behavior exhibited by $\Lambda$.

Our point of departure in [Calini and Langer 03] was to represent elements of $\Lambda$ via Schwarz functions; we recall from [Davis 74] that the Schwarz function of an analytic curve $\Gamma$ is the unique holomorphic function $S(z)$ for which $\Gamma$ is the locus of the equation $\bar{z}=S(z)$. A key point was that Schwarz functions could be viewed as symmetric elements [Loos 69$]$ in the abstract setting of a group with involution $(G, \sigma)$, and that symmetric-space formalism could subsequently be invoked. (Here, $G$ consists of conformal maps, $\sigma(g)(z)=\overline{g(\bar{z})}$, and the operations of composition, inversion, and involution are only locally defined.) Further, the use of Schwarz functions yielded concrete analytic expressions for basic geometric objects and operations that were seen in [Calini and Langer 03] to be remarkably tractable.

In particular, a compact formula for the canonical connection on $\Lambda$ was derived, and this led to a simple geodesic equation - a conformally invariant, quadratic, second-order PDE for a time-dependent Schwarz function $S(t, z)$, implicitly describing an evolving analytic curve $\Gamma_{t}$. Moreover, the latter equation was shown to coincide with a continuous limit of iterated Schwarzian reflection of analytic curves. Finally, such motion was reduced, locally, to the flow of a holomorphic vector field $W=w(z) \frac{\partial}{\partial z}$, and data associated with the dual meromorphic differential $\omega=d z / w(z)$ were seen to determine conformal-geometric properties of the evolving curve.

In the present paper, we continue to develop major themes of Schwarz reflection geometry initiated in [Calini and Langer 03], systematically addressing some of the main problems posed there. For example, we obtain a comprehensive local description of all geodesics, up to conformal equivalence, near a given initial curve $\Gamma_{\circ}$, and generate representative examples. More specifically, by considering the action of the group of analytic circle diffeomorphisms $H=\operatorname{Diff}_{+}^{\omega}\left(S^{1}\right)$ on (normal) analytic vector fields $W$ along $S^{1}$, we obtain the following main result on short-time behavior of geodesics departing from $\Gamma_{\circ}=S^{1}$ :

For $W=w(z) \frac{\partial}{\partial z} \in T_{\Gamma_{\circ}} \Lambda$, the geodesic departing from $\Gamma_{\circ}$ with velocity $W$ is representable in the form $\exp (t W)=h(\exp (t V))$, for small $|t|$, with $h \in H$ and rational vector field

$$
V=\frac{a_{m} z^{m}+\cdots+a_{0}}{b_{n} z^{n}+\cdots+b_{0}} \frac{\partial}{\partial z},
$$

which can be constructed explicitly using meromorphic data of $\omega=d z / w(z)$, the dual differential to $W$.

The result is very simple for the special case of a vector field $W$ that is nonvanishing along $S^{1}$ : $W$ is equivalent to a uniform field $\frac{i}{c} \frac{\partial}{\partial \theta}$, with real parameter $c=\frac{1}{2 \pi} \int_{S^{1}} i \omega$ characterizing the orbits $H \frac{i}{c} \frac{\partial}{\partial \theta} \simeq H / S^{1}$. The curves constituting the maximal geodesic $\Gamma_{t}=\exp (t W)$ for $|t|<m$ foliate a singular ring domain $\mathcal{D}$ in the extended complex plane. Allowing $W$ to vary over any given orbit, the maximal geodesics $\exp (t W)$ define a geodesic foliation of $\Lambda_{ \pm} \subset \Lambda$, the space of all simple closed analytic curves in the extended complex plane disjoint from $S^{1}$.

Such conclusions may be drawn readily from the theory of conformal mapping and moduli of ring domains, which also can be used to derive information about the time $m$ required for a given such geodesic $\exp (t W)$ to run out of $\Lambda$. In terms of locations of singularities of the (normalized) vector field $\hat{W}$, we obtain a sharp upper bound on $m(\hat{W})$; see Proposition 5.3. The sharpness of our estimate, given by a ratio of elliptic integrals, follows from the solution to an extremal problem that is well known in quasiconformal mapping theory.

Turning to the general case, $W \in T_{\Gamma_{\circ}} \Lambda$, it is natural to draw the connection between $\exp (t W)$ and the established circle of ideas involving meromorphic flows, trajectories of quadratic differentials, and foliated flat geometries (see, e.g., [Muciño-Raymundo 02]); these ideas are incorporated into our examples, which are integral to the development of the theoretical results presented here. However, such standard methods do not appear to give one a handle on such questions as, which curves $\Gamma \in \Lambda$ intersecting $\Gamma_{\circ}$ lie in the image of the exponential map at $\Gamma_{0}$ ?

On the other hand, the structure of the Lie algebra $\mathfrak{g}=\mathfrak{h} \oplus i \mathfrak{h}$ of analytic vector fields along $S^{1}$ is directly relevant to questions of local geometry of $\Lambda$. For example, the bracket relations for the Witt algebra easily imply that $\Lambda$ does not have pseudo-Riemannian structure (Proposition 6.1). Further, the proof of the main result (see Theorem 7.3) is related to the classification of 
adjoint orbits of $H$. To be precise, the following result (see Theorem 10.2) provides the basis for explicit construction of normal forms for the vector fields $W \in T_{\Gamma_{\circ}} \Lambda$ and locally representative examples of geodesics $\exp (t W)$ (using data $\mu_{j}, \nu_{j}, \sigma_{j}, \mathcal{I}_{k}, \mathcal{I}$ to be described later):

(i) Two real meromorphic differentials on $S^{1}$ are equivalent if and only if they have identical orderresidue-polarity-period data, $\mu_{j}, \nu_{j}, \sigma_{j}, \mathcal{I}_{k}, \mathcal{I}$, for suitable counterclockwise orderings of singularities.

(ii) For such differentials with no zeros on $S^{1}$, all equivalence classes are represented by restriction to $S^{1}$ of rational differentials on $\hat{\mathbb{C}}=\mathbb{C} \cup\{\infty\}$, since the latter achieve arbitrary data $\mu_{j}, \nu_{j}, \sigma_{j}, \mathcal{I}$, subject only to the polarity constraints and, when there are no poles, $\mathcal{I} \neq 0$.

(iii) The adjoint orbits $\operatorname{Ad}_{H} A$ are characterized by data of the dual meromorphic differentials as in item (ii); in particular, each is represented by restriction to $S^{1}$ of a rational vector field on $\hat{\mathbb{C}}$.

Our concrete examples and graphical representations ${ }^{1}$ of geodesics not only serve to illustrate the above results, but also raise questions of interpretation that need to be addressed for further development of the subject. In particular, in various ways, our examples bring focus to the question, how is one to address the lack of an actual Lie group $G=H_{\mathbb{C}}$ with Lie algebra $\mathfrak{g}$ ?

Thus, with a view toward a more global theory, we explore issues of apparent multivaluedness and push analytic continuation beyond the point at which our (provisional) notions of Schwarz reflection geometry break down. In this context, Example 4.1 exposes the relation to trajectory structure of quadratic differentials and foliated flat geometries as not entirely clear-cut; Example 4.3 displays geodesics with topological transitions involving singular and multicomponent curves $\Gamma \notin \Lambda$ (but see Remark 4.4 for an explanation of all other graphical examples); Example 5.1 admits interpretation as a closed geodesic foliating a genus-one Riemann surface (Remark $5.2)$; Example 6.2 describes geodesics $\exp \left(t W_{n}\right)$ determined by Witt algebra basis elements via "covering-map symmetries" $h \notin H$ reflected in the structure of $\mathfrak{h}$.

Some final notes on the contents and organization of the paper may be helpful. Elements of Schwarz reflection geometry introduced in [Calini and Langer 03] are briefly

\footnotetext{
${ }^{1}$ Color versions, which better illustrate certain features of the geodesics, are available online (http://www.case.edu/artsci/math/ langer/research/papers/srgII.pdf).
}

reviewed and extended in Sections 2 and 3. We begin by relativizing the notion of Schwarz function, allowing an arbitrary analytic curve to be used as base point $\Gamma_{\circ} \in \Lambda$. Regarding the resulting functions $S=S_{\Gamma_{\circ}}$ as local coordinates on $\Lambda$, we discuss transformation rules, invariance properties, and variations of curves $\Gamma_{t}$ and corresponding Schwarz functions $S(t, z)$. We give equations for the canonical connection on $(\Lambda, \cdot)$, geodesics $(3-1)$, and their canonical parametrization (3-5). In Section 4 we describe the connection between geodesics in $\Lambda$ and trajectories of quadratic differentials. The main contents of Sections 5 through 7 on ring domains, the isotropy representation $\lambda_{*}$, and geodesics generated by rational vector fields have already been mentioned above. The last of these sections presents only the "constructive part" of the proof of Theorem 7.3, since it directly supports examples; the underlying theoretical framework and results are deferred to Sections 8 through 10, so as not to interrupt the main flow of ideas of Schwarz reflection geometry. Specifically, in Section 8 we discuss analytic diffeomorphisms of the circle; in Section 9, we consider real meromorphic differentials on $S^{1}$, and describe their invariants with respect to the action of $H$ by pullback; finally, Section 10 treats the equivalence problem for such differentials and completes the proofs of Theorems 7.3 and 10.2.

\section{ANALYTIC JORDAN CURVES AND THEIR SCHWARZ FUNCTIONS}

The next paragraphs introduce notation and recall some facts about real analytic curves; further details may be found in [Ahlfors 53, Chapter 5, Section 1.6]. An analytic arc or curve $\Gamma=\gamma([a, b]) \subset \mathbb{C}$ has a parametrization $\gamma(t)=x(t)+i y(t)$ by real analytic functions $x, y:[a, b] \rightarrow$ $\mathbb{R}$. One normally assumes $\Gamma$ to be regular in the sense that $\gamma$ may be chosen to have nonvanishing velocity $\gamma^{\prime}(t) \neq 0$. For a Jordan arc, $\gamma$ is one-to-one, and $\Gamma$ may be regarded as the image of $I=[a, b]$ under a conformal map defined by analytic continuation of $\gamma$ to a symmetric neighborhood $U=U^{*} \subset \mathbb{C}$ of $I$; here, $U^{*}=\left\{z^{*}=\bar{z}: z \in U\right\}$. More specifically, one may replace $t$ by $t+i \tau$ in each local power series representation

$$
\gamma=\sum_{j=0}^{\infty} a_{j}\left(t-t_{0}\right)^{j}, \quad t_{0} \in I
$$

and restrict to $\left|t-t_{0}\right|<r$ for sufficiently small $r>0$, to obtain such a conformal extension $\gamma: U \rightarrow \mathbb{C}$.

In the case of a closed Jordan curve, we regard $\Gamma$ instead as the image of the unit circle under a conformal 
mapping $\gamma: U \rightarrow \mathbb{C}$ defined on a symmetric neighborhood of $S^{1}$. That is, $U=U^{*} \subset \mathbb{C} \backslash\{0\}$, where $U^{*}=\left\{z^{*}=1 / \bar{z}: z \in U\right\}$. It will not cause confusion to use the same notation $b(z)=z^{*}$ for the conjugate (or reflection) of a point $z$ with respect to $\mathbb{R}$ or $S^{1}$, depending on which of the two cases is considered; likewise for other notation just introduced.

Now consider the image $V=\gamma(U)$ and let $\beta=\beta_{\Gamma}$ : $V \rightarrow V$ be defined by

$$
\beta(z)=\gamma\left(\left(\gamma^{-1}(z)\right)^{*}\right)=\gamma \circ b \circ \gamma^{-1}(z) .
$$

Then $\beta$ is antiholomorphic, and restricts to the identity mapping on $\Gamma$. In fact, these two properties locally characterize $\beta$; thus, at each $z_{0} \in \Gamma$, the germ of Schwarzian (or antiholomorphic) reflection in $\Gamma$ is well defined by the above formula. Likewise, $\beta=\beta^{-1}$ inherits involutivity from the basic reflection $b$.

Reflection of points in $\Gamma$ induces conjugation (or reflection) of functions with respect to $\Gamma$. That is, a holomorphic function with suitable domain and range yields a new holomorphic function by the operation $f \mapsto \sigma(f)=$ $\beta \circ f \circ \beta$. Most familiar is the case $\Gamma=\mathbb{R}$, allowing any function to be conjugated; if

$$
f(z)=\sum_{j=0}^{\infty} a_{j}\left(z-z_{0}\right)^{j}
$$

is holomorphic on $U$, then

$$
\sigma(f)(z)=\overline{f(\bar{z})}=\sum_{j=0}^{\infty} \bar{a}_{j}\left(z-\bar{z}_{0}\right)^{j}
$$

is holomorphic on $U^{*}$. When $f$ happens to take real values on the real axis, $f$ is self-conjugate $(\sigma(f)=f)$, and the Schwarz symmetry principle holds: $w=f(z)$ maps a symmetric pair $\left\{z, z^{*}\right\}$ to a symmetric pair $\left\{w, w^{*}\right\}$. (The symmetry principle may be regarded as the basis of the well-known Schwarz reflection principle, which we will require in Section 5; the holomorphic extension provided by this classical result, as usually stated, is self-conjugate by construction.)

Similar remarks apply to the case $\Gamma=S^{1}$. For a general analytic curve $\Gamma$, conjugation may be applied only to a smaller class of functions, since $\beta$ itself is only locally defined. However, the allowable class includes at least holomorphic functions $f$ that are defined, and sufficiently close to the identity, along $\Gamma$; this observation will suffice to make sense of our constructions below, which apply to the following situation. An arbitrarily chosen base curve $\Gamma_{\circ}$ will play the role of the initial reflecting curve, while $\Gamma$ will now denote a second, nearby curve. Such a curve may be parametrized by $\gamma: \Gamma_{\circ} \rightarrow \Gamma$, which is close to the identity on $\Gamma_{\circ}$ and extends to a conformal mapping that may be allowed to play the role of $f$, the function to be conjugated. We note that $\gamma$ will never be self-conjugate, that is, assuming that $\Gamma$ is close but not identical to $\Gamma_{\circ}$. The context just described is thus complementary to that of the Schwarz symmetry (or reflection) principle, where the operation $f \mapsto \sigma(f)$ is in itself uninteresting!

The preceding paragraphs pave the way for the introduction of Schwarz functions relative to a base curve $\Gamma_{0}$. The general definition is also guided by a simple symmetric space recipe: If $G$ is a Lie group with involution $\sigma$, the formula

$$
p \cdot q=p \sigma(q) p=p q^{-1} p
$$

defines a symmetric-space multiplication (in the sense of [Loos 69]) on the subset of symmetric elements

$$
G_{\sigma}=\left\{\sigma(g) g^{-1}: g \in G\right\} \subset G .
$$

In particular, Schwarzian reflection across an embedded analytic curve $\Gamma_{\circ}$ formally defines such an involutive automorphism $\sigma$ with respect to composition in the pseudogroup $G$ consisting of local conformal maps, and one may therefore expect a symmetric-space geometry to result as above.

Specifically, to a conformally parametrized curve $\gamma$ : $\Gamma_{\circ} \rightarrow \Gamma$ we associate a holomorphic function $S=S_{\Gamma_{\circ} \Gamma}$ defined near $\Gamma$, the Schwarz function of $\Gamma$ relative to $\Gamma_{\circ}$ :

$$
S=\sigma(\gamma) \circ \gamma^{-1}
$$

(Fixing the real axis as base curve $\Gamma_{\circ}=\mathbb{R}$ yields the standard notion of Schwarz function as defined in [Davis 74]; this was the definition used in [Calini and Langer 03].) We list a few properties of $S$ that follow as formal consequences of (2-1) (valid under suitable assumptions on domains, etc.):

(a) $S$ does not depend on the parametrization $\gamma$ of $\Gamma$;

(b) $S_{\Gamma_{\circ} \Gamma_{\circ}}=$ Id;

(c) $S^{-1}=\sigma(S)$;

(d) $\beta \circ S$ gives antiholomorphic reflection across $\Gamma$;

(e) given $S$, the equation $\beta(z)=S(z)$ implicitly defines the analytic curve $\Gamma$. 
Since a curve (near $\Gamma_{\circ}$ ) may thus be recovered from its Schwarz function (relative to $\Gamma_{\circ}$ ), we will speak informally of "the curve $S(z)$ " when making local arguments.

Next, we note that if $S_{1}(z)$ and $S_{2}(z)$ are two such curves, the antiholomorphic reflection of $S_{1}$ in $S_{2}$ is given by multiplication of Schwarz functions:

$$
S_{3}=S_{2} \cdot S_{1}=S_{2} \circ S_{1}^{-1} \circ S_{2} .
$$

Also, left multiplication by $P, Q \mapsto l_{P} Q=P \cdot Q$ satisfies the formal properties defining a symmetric-space multiplication in the sense of [Loos 69]: for each $P \in \Lambda$, $l_{P}: \Lambda \rightarrow \Lambda$ is an involutive automorphism of $\Lambda$ with isolated fixed point $P$. It will be seen below that in fact, $l_{P}$ is the geodesic symmetry with respect to $P$ in the standard symmetric-space sense. The automorphism $Q \mapsto l_{\text {Id }} Q=Q^{-1}$ corresponding to reversal of geodesics through the base curve will appear more concretely below.

The canonical left action $\lambda: G \times G_{\sigma} \rightarrow G_{\sigma}$ is given by the automorphisms $p \mapsto \lambda_{g}(p)=\sigma(g) p g^{-1}$. In the present context, if $S$ is the Schwarz function of $\Gamma$ and $g(z)$ is a conformal map defined near $\Gamma$, then the Schwarz function of the image curve $g(\Gamma)$ is given by

$$
\lambda_{g}(S)=\sigma(g) \circ S \circ g^{-1},
$$

and $\lambda_{g}\left(S_{2} \cdot S_{1}\right)=\lambda_{g}\left(S_{2}\right) \cdot \lambda_{g}\left(S_{1}\right)$ holds. The local action is transitive on simple closed analytic curves, since a conformal map $g(z)$ between interiors of such curves extends conformally to the boundary. We will be especially interested in $g=h$ belonging to the diffeomorphism group $H$ of the base curve, in which case $\sigma(h)=h$ and the action reduces to ordinary conjugation. Determining the orbits of this restricted action is far from trivial.

Change of base curve rules require more explicit notation. For example, if $P, Q, R$ are three analytic curves and $S_{P Q}, S_{P R}$ are the Schwarz functions of $Q$ and $R$ relative to $P$, and $S_{Q R}$ is the Schwarz function of $R$ relative to $Q$, then

$$
S_{P R}=S_{P Q} \circ S_{Q R}
$$

In particular, $S_{P Q} \circ S_{Q P}=S_{P P}=$ Id. Also, $(2-3)$, $(2-4)$ give

$$
S_{g(P) g(Q)}=g \circ S_{P Q} \circ g^{-1}
$$

for $g(z)$ a conformal map defined near $P, Q$.

Concrete computations are simplest with base curve $\Gamma_{\circ}=\mathbb{R}$. Even for $\Gamma_{\circ}=S^{1}$, it pays to take advantage of the above rules rather than compute directly. For instance, setting $P=\hat{\mathbb{R}}, Q=S^{1}$ in $(2-4)$, we have $S_{P Q}(z)=S_{Q P}(z)=1 / z$, and the Schwarz functions of a third curve $R$ relative to $P, Q$ are reciprocals:
$S_{Q R}(z)=1 / S_{P R}(z)$. Similarly, direct verification of the following proposition is easiest in the case $\Gamma_{\circ}=\mathbb{R}$, from which the general case follows by an application of $(2-5)$ :

Proposition 2.1. For a fixed base curve $\Gamma_{\circ}$, consider a time-dependent, parametrized analytic curve $x \mapsto \gamma(t, x)$, $x \in \Gamma_{\circ}$, and corresponding Schwarz function $S(t, z)$ relative to $\Gamma_{\circ}$. Letting $n(t, x)$ denote a unit normal vector field along the curve $\Gamma_{t}=\gamma\left(t, \Gamma_{\circ}\right)$, the normal variation field $\dot{\gamma}^{n}=\langle n, \dot{\gamma}\rangle n$ of $\gamma$ is given in terms of $t$ and $z$-derivatives of $S$ by

$$
\dot{\gamma}^{n}(t, x)=-\frac{1}{2} \frac{\dot{S}(t, \gamma(t, x))}{S^{\prime}(t, \gamma(t, x))} .
$$

Thus, if $\gamma(0, x)=x$, the initial variation of $S(t, z)$ may be regarded as a normal vector field along $\Gamma_{\circ}$; that is, $\dot{S}(0, x)=-2 \dot{\gamma}^{n}(0, x), x \in \Gamma_{\circ}$.

Note: here we are using the identification $a+i b \leftrightarrow$ $(a, b) \in T_{\left(x_{0}, y_{0}\right)} \mathbb{R}^{2}$; we will continue to do so throughout Section 3, after which we will adopt complex vector field notation $w(z) \frac{\partial}{\partial z}$ and related formalism.

\section{GEODESICS IN $\Lambda$}

In differential geometry the notion of geodesic may be regarded as one of the most basic consequences of geometric structure. This is especially true in Riemannian geometry, where a geodesic may be described, locally, as the shortest path joining a given pair of points $p, q \in M$. However, other geometric settings do not allow geodesics to be characterized quite so simply. For instance, in the pseudo-Riemannian case, an indefinite form $\langle X, Y\rangle$ on each tangent space $T_{p} M$ defines the geometry, and consequently "shortest paths" need not exist. Actually, one may continue to take a variational approach to geodesics in this case by invoking the more general least-action principle (as it applies in physics): the integral

$$
\int_{a}^{b}\left\langle\frac{d \alpha}{d t}, \frac{d \alpha}{d t}\right\rangle d t
$$

defined on a space of paths $\alpha:[a, b] \rightarrow M$ joining given nearby points $p=\alpha(a)$ and $q=\alpha(b)$ is required to be stationary (critical), but is not necessarily minimized.

Indeed, the latter case arises in our context, for it was seen in [Calini and Langer 03] that the space of circles in the Riemann sphere may be regarded as a three-dimensional symmetric subspace $\Lambda^{3}$ of the space of analytic curves $\Lambda$ and that this geometry on $\Lambda^{3}$ is 
pseudo-Riemannian. The description of geodesics in $\Lambda^{3}$ consequently breaks into spacelike, lightlike, and timelike cases, according to whether the velocity vector $X=\frac{d \alpha}{d t}$ satisfies $\langle X, X\rangle>0,\langle X, X\rangle=0$, or $\langle X, X\rangle<0$. On the more concrete level, the corresponding family of "continuously reflected" circles has two, one, or zero common points of intersection.

The discussion of $\Lambda^{3}$ in [Calini and Langer 03] led naturally to the question whether the geometry of the whole space $\Lambda$ likewise admitted a metric structure of some type. A negative answer is given in Section 6, based on the symmetries of $\Lambda$ already described above. Such a result is hardly surprising; one may compare with classical, nonmetric geometries, such as projective, conformal or "school" geometries (similarities being the underlying symmetries of the latter). We will still be able to regard geodesics as basic objects in our geometry, where the intuition of "shortest path" should perhaps be replaced by "straightest path," as measured via the notions of parallel transport or connection, which we now proceed to introduce in our context.

According to the general theory of [Loos 69], a symmetric-space multiplication directly induces an affine connection $\nabla$, the canonical connection. In the present situation, this connection may be expressed as

$$
\nabla_{X} Y=\bar{\nabla}_{X} Y-\frac{1}{2 S_{z}}\left(X_{z} Y+Y_{z} X\right)
$$

using the local identification of $\Lambda$ with Schwarz functions relative to a fixed base curve $\Gamma_{\circ}$. We will not require this formula - it is derived in [Calini and Langer 03] using $\Gamma_{\circ}=\mathbb{R}$-but we note that it inherits invariance properties (with respect to change of base curve and the canonical left action $\lambda$ ) from those of the multiplication rule $(2-2)$.

As a consequence, one obtains an invariant geodesic equation:

$$
0=\nabla_{\dot{S}} \dot{S}=\ddot{S}-\frac{\dot{S}}{S^{\prime}} \dot{S}^{\prime}
$$

In [Calini and Langer 03] this equation is also interpreted as a continuous limit of iterated Schwarzian reflection, obtained by allowing an initial pair of curves to approach each other. For the sake of "physical intuition," it should be noted also that such a geodesic equation is of second order in time (as usual) and in this sense resembles Newton's law $F=m a$, even if it may not be a consequence of a least-action principle.
Since the above PDE may be written $\partial_{t}\left(\dot{S} / S^{\prime}\right)=0$, a solution $S(t, z)$ must satisfy a first-order linear PDE:

$$
\frac{\dot{S}(t, z)}{S^{\prime}(t, z)}=-2 w(z)
$$

Here, $w(z)$ is taken to be holomorphic on a neighborhood of $\Gamma_{0}$. If we change the base curve to $\Gamma_{1}$ and write $S_{1}=S_{\Gamma_{1} \Gamma_{0}}$, then the new Schwarz function $\tilde{S}(t, z)=$ $S_{1} \circ S(t, z)$ satisfies again $\dot{\tilde{S}}(t, z) / \tilde{S}^{\prime}(t, z)=-2 w(z)$. (As a special case of this invariance property, the base curves $\Gamma_{\circ}=\mathbb{R}$ and $\Gamma_{\circ}=S^{1}$ yield reciprocal pairs of solutions $S, 1 / S$.) On the other hand, if we leave the base curve alone but conformally transform the evolving curve by $g(z)$, we find that $\tilde{S}=\lambda_{g}(S)=\sigma(g) \circ S \circ g^{-1}$ satisfies $\dot{\tilde{S}}(t, z) / \tilde{S}^{\prime}(t, z)=-2 \tilde{w}(z)$, where $\tilde{w}$ is given by the transformation rule

$$
\tilde{w}(z)=\lambda_{g *} w(z)=g^{\prime}\left(g^{-1}(z)\right) w\left(g^{-1}(z)\right),
$$

and (3-1) continues to hold.

Equation (3-2) is written with the factor -2 so that according to Proposition 2.1, $w(\gamma(t, x))$ describes the normal velocity field of the corresponding moving curve $\Gamma_{t}=\gamma\left(t, \Gamma_{\circ}\right)$ :

$$
\dot{\gamma}^{n}(t, x)=w(\gamma(t, x)), x \in \Gamma_{\circ} .
$$

Remark 3.1. Comparing (2-6), (3-2), and (3-4), one arrives at the following important conclusion: What distinguishes geodesic motions among the more general variations representable via time-dependent Schwarz functions is that the former are generated by time-independent holomorphic vector fields.

This interpretation may be realized more concretely. First observe that if we fix the initial condition $S(0, z)=$ $z$, then $(3-2)$ is but a differentiated version of the equation $S(t+u, z)=S(t, S(u, z))$ describing a oneparameter group $S(t, z)$. In particular, a time-dependent Schwarz function representing a geodesic $\Gamma_{t}$ departing from $\Gamma_{\circ}$ at $t=0$ satisfies $S(-t, z)=S^{-1}(t, z)=$ $\sigma(S)(t, z)$ (and the automorphism $S \mapsto \sigma(S)$ clearly reverses geodesics through $\Gamma_{\circ}$ as claimed earlier). Now let $\theta \mapsto x(\theta) \in \Gamma_{\circ}$ be a parametrization of $\Gamma_{\circ}$ with real parameter $\theta$, and set $\zeta(t, x)=S(-t / 2, x)$ for $x=x(\theta) \in \Gamma_{\circ}$. Using the group law one obtains

$$
\begin{aligned}
S(t, \zeta(t, x)) & =S(t / 2, x)=\sigma(S)(-t / 2, x)=\beta(S(-t / 2, x)) \\
& =\beta(\zeta(t, x)),
\end{aligned}
$$

precisely the condition for $\zeta(t, x)$ to lie on $\Gamma_{t}$. In fact, $\zeta(t, x(\theta))$ parametrizes $\Gamma_{t}$ by normal motion, for 
$(3-2)$ implies $\dot{\zeta}(t, x)=\zeta^{\prime}(t, x) w(x)$, and hence the ratio $\partial_{t} \zeta / \partial_{\theta} \zeta=w / x^{\prime}$ is imaginary, being a ratio of normal and tangent vectors along $\Gamma_{\circ}$. It follows that $\dot{\zeta}^{n}(t, x)=$ $\dot{\zeta}(t, x)$. To summarize, we have the following result.

Proposition 3.2. Let $\Gamma_{t}$ be a geodesic emanating from $\Gamma_{0}=\Gamma_{\circ}$, with Schwarz function $S(t, z)$ satisfying $(3-2)$ and $S(0, z)=z$. Then $\Gamma_{t}$ has canonical parametrization

$$
\zeta(t, x)=S(-t / 2, x), x \in \Gamma_{\circ}
$$

describing normal motion of $\Gamma_{t}=\zeta\left(t, \Gamma_{\circ}\right)$. Further, $t \mapsto$ $\zeta(t, x)$ satisfies the following ODE initial value problem with parameter $x \in \Gamma_{\circ}$ :

$$
\dot{\zeta}(t, x)=w(\zeta(t, x)), \quad \zeta(0, x)=x .
$$

We will also use the notation $\exp =\exp _{\circ}$ for the exponential map based at $\Gamma_{\circ}$; namely, $\Gamma_{t}=\zeta\left(t, \Gamma_{\circ}\right)=\exp (t w)$ is the unique geodesic with initial point $\Gamma_{\circ}$ and initial tangent $w(z)$. Of course, $\exp (t w)$ is also given by the time-dependent Schwarz function $S(t, z)=\zeta(-2 t, z)$, the solution to the parametrized ODE

$$
\dot{S}(t, z)=-2 w(S(t, z)), \quad S(0, z)=z .
$$

Here we have replaced $x \in \Gamma_{\circ}$ by $z$ in a neighborhood $U$ of $\Gamma_{\text {。 }}$ where $S$ is holomorphic (say, in view of analytic dependence of solutions on parameters). Passing from (3-2) to the latter ODE corresponds to the method of characteristics.

\section{HOLOMORPHIC FLOWS AND TRAJECTORIES OF DIFFERENTIALS}

In the previous section it was notationally expedient to write $w(z)$ in place of $W=w(z) \partial_{z}$ - essentially using local coordinates to identify a holomorphic function with a holomorphic vector field. Now the operator notation $W=w(z) \partial_{z}$ becomes much more appropriate as we consider duality and other invariant notions of geometric and Lie-algebraic structure. Though the complex vector field formalism is perhaps unintuitive, it also helps distinguish the planar flows we wish to consider from the ideal fluid flows that have been associated commonly with holomorphic functions since the nineteenth century, when Riemann and others considered physical applications and interpretations of function theory. (The difference between the two types of planar flows will be mentioned below, and [Calini and Langer 03] includes a fuller explanation.)
To begin, we identify $W=\frac{1}{2}(u+i v)\left(\partial_{x}-i \partial_{y}\right)$ as needed with its "real part" $X=\Re(W)=u \partial_{x}+v \partial_{y}$; the "imaginary part" $Y=\Im(W)=-v \partial_{x}+u \partial_{y}$ may be computed as $Y=J X$; hence one may always recover $W=\frac{1}{2}(X-i Y)$. Thus, for example, we will associate to $W$ the real planar flow defined by $X$ and its conjugate flow given by $Y$. The flow generated by $W$ with complex time $\tau=t+i s$ incorporates both of these. (Our terminology and notation here mostly follow that of [MuciñoRaymundo 02]; see also [Kobayashi and Wu 83, pp. 7071] for standard identifications in the holomorphic vector field context.)

Via duality $W=w(z) \partial_{z} \leftrightarrow \omega=d z / w(z)$, one may describe the trajectories of $X$ (and those of $Y$ ) in terms of the corresponding differential

$$
\omega=\frac{u-i v}{u^{2}+v^{2}}(d x+i d y)
$$

or quadratic differential $p=\omega^{2}=d z^{2} / w^{2}$ : the horizontal trajectories satisfy the equation

$$
0=\Im(\omega)=\frac{u d y-v d x}{u^{2}+v^{2}},
$$

i.e., $p>0$, while the vertical trajectories satisfy

$$
0=\Re(\omega)=\frac{u d x+v d y}{u^{2}+v^{2}},
$$

i.e., $p<0$. We note that the corresponding (incompressible, irrotational) "fluid flows" $X /|X|^{2}, Y /|Y|^{2}$ determine the same orthogonal pair of singular foliations (streamlines and equipotentials). Unlike the latter fields, however, $X$ and $Y$ commute, as a consequence of the Cauchy-Riemann equations for $u, v$. By the same token, $\omega$ is closed, with local potential

$$
\phi(z)=U(x, y)+i V(x, y)=\int_{z_{0}}^{z} \omega
$$

defining a holomorphic coordinate (natural parameter) $\tau=t+i s=\phi(z)$ near $z_{0}$, a regular point. The potential $\phi$ maps small "curvilinear rectangles" bounded (and foliated) by $U$ and $V$ level sets onto Cartesian rectangles $t_{1}<t<t_{2}, s_{1}<s<s_{2}$, and gives local canonical forms $W=\partial_{\tau}, \omega=d \tau, p=d \tau^{2}$.

Returning to geodesics $\Gamma_{t}=\zeta\left(t, \gamma_{\circ}\right)=\exp (t W)$ in $\Lambda$, the velocity field of the canonical parametrization $\zeta$ might now be properly written $X=\Re(W)$-in place of $w$ in (3-6)-but we will simply display $W=w \partial_{z}$ in our examples below. We compute $\zeta(\tau, z)$ by solving

$$
\omega=d \zeta / w(\zeta)=d \tau, \zeta(0, z)=z .
$$


In terms of local potential $\phi(z)$ as above, we have the implicit solution

$$
\phi(\zeta(\tau, z))=\phi(z)+\tau
$$

valid in the neighborhood of a regular point $z_{0}$, for small $\tau$. We may subsequently specialize to real time $\tau=t$ and $z=x \in \Gamma_{\circ}$ to obtain the canonical parametrization $\zeta(t, x)$. For fixed $t_{0}$, the curve $\Gamma_{t_{0}}=\zeta\left(t_{0}, \Gamma_{\circ}\right)$ belongs locally to a vertical trajectory of $p=\omega^{2}$, and satisfies $U=U_{0}=$ constant. In fact, using $\tau=t$ and $z=x \in$ $\Gamma_{\circ}$, the real part of $(4-2)$ becomes $U(\zeta(t, x))=U(x)+$ $t=U_{0}+t$ (note that $Y$ is tangent to $\Gamma_{0}$, and $d U(Y)=$ $\Re(\omega)(\Re(-i W))=0)$.

On the other hand, a point starting at $x_{0} \in \Gamma_{\circ}$ follows a horizontal trajectory $\zeta\left(t, x_{0}\right)$ satisfying $V=$ const. The conjugate geodesic to $\Gamma_{t}$, denoted by $\star \Gamma_{s}=\zeta\left(i s, \star \Gamma_{\circ}\right)$, comprises the latter curves and may be associated with $\star \omega=-i \omega$ and $J W$; it is determined locally by $\Gamma_{t}$ and initial orthogonal curve $\star \Gamma_{\circ}$. With respect to this curve, $\star \Gamma_{s}$ has Schwarz function $\star S(s, z)=S(i s, z)$.

In simple examples, such computations will be seen to have more or less global interpretations - even in the presence of singular points - by due consideration of multivaluedness, etc. For general purposes, however, the above description is inadequate, and one requires a suitable theory of canonical forms. There is such a local canonical form result for a meromorphic quadratic differential $p=P(z) d z^{2}$ near a singular point [Strebel 84]; but we do not invoke such results from the standard theory, since our context is rather special. For one thing, it will suffice for our purposes to restrict to orientable quadratic differentials $p=\omega^{2}$ (see, however, Remark 5.2). Further, we are primarily concerned with nonvanishing meromorphic differentials $\omega$ defined (and real or imaginary) along $\Gamma_{\mathrm{o}}$, since we are currently assuming $W$ holomorphic (and tangential or normal) along $\Gamma_{\circ}$. Nor is our situation covered as a special case of the above-mentioned local normal form, since we will require a description of $\omega$ near $\Gamma_{\circ}$ not merely in the vicinity of a single point $z_{0} \in \Gamma_{\circ}$. In Theorem 10.2, we give the relevant statement (and direct proof).

It will nevertheless be useful to recall one of the basic ideas in the standard theory; namely, issues of (global as well as local) equivalence for quadratic differentials may be discussed in terms of isometric equivalence with respect to the (singular) Riemannian metric

$$
g=|P(z)| d z d \bar{z}=\frac{d x^{2}+d y^{2}}{u^{2}+v^{2}}=\phi^{*}\left(d x^{2}+d y^{2}\right) .
$$

Near a regular point, the commuting fields $X, Y$ are orthonormal with respect to this flat metric, which is the pullback of the Euclidean metric by the local isometry $\phi$. The vector fields $X$ and $Y$ generate local isometries, and their trajectories are unit-speed geodesics. The lengths of curves $\mathcal{L}=\int_{\gamma}|P|^{1 / 2}|d z|$ and areas of regions $\mathcal{A}=\iint_{\mathcal{R}}|P| d x d y$ are useful, for example, in discussing moduli of ring domains. Our concrete examples will include geometric descriptions along such lines, with everything expressed in terms of $\omega: p=\omega^{2}, g=\omega \bar{\omega}$, $\mathcal{L}=\int_{\gamma}|\omega|$, etc.

Example 4.1. (Circles.) The unoriented circles in the Riemann sphere form a three-dimensional subspace $\Lambda^{3} \subset \Lambda$ in which the multiplication reduces to the usual circle inversion. The space $\Lambda^{3}$ is (double-covered by) a generalized sphere in Lorentzian four-space, and the three isometry classes of geodesics in $\Lambda^{3}$ have timelike, spacelike, or lightlike initial velocity vectors, corresponding to $W=w(z) \partial_{z}$ having no zeros, two zeros, or a double zero on $\Gamma_{\circ}=S^{1}$ (as discussed in [Calini and Langer 03] using $\left.\Gamma_{\circ}=\mathbb{R}\right)$.

Here we consider geodesics in $\Lambda^{3}$ to illustrate conformal invariants and flat geometries. It suffices to look at geodesics departing from the base curve $\Gamma_{\circ}=S^{1}$ with the following initial velocities:

$$
\begin{aligned}
& W_{-}=-i \partial_{\theta}=z \partial_{z}, \\
& W_{+}=-i \sin \theta \partial_{\theta}=\frac{1}{2 i}\left(z^{2}-1\right) \partial_{z}, \\
& W_{0}=-i(1-\cos \theta) \partial_{\theta}=-(z-1)^{2} \partial_{z} .
\end{aligned}
$$

(We note that $W_{-}, W_{+}, W_{0}$ generate the algebra $\mathfrak{s l}(2, \mathbb{C})$, with Cartan-Killing form satisfying $\operatorname{sgn}\left(\left\langle W_{\sigma}, W_{\sigma}\right\rangle\right)=\sigma$.)

(a) $W_{-} \leftrightarrow \omega=d \log z$. With $\phi=\log z,(4-2)$ gives $\zeta\left(t, e^{i \theta}\right)=e^{t+i \theta}$. Then $\Gamma_{t}=C_{e^{t}}$ is the "expanding circles" foliation of

$$
\mathbb{C}^{\times}=\mathbb{C} \backslash\{0\}: U=\ln |z|=t .
$$

We note that $\Gamma_{t}$ has Schwarz function $S(t, z)=e^{-2 t} z$ (with respect to $\Gamma_{\circ}=S^{1}$ ). The conjugate geodesic $\star \Gamma_{s}$ has Schwarz function $\star S(s, z)=e^{-2 i s} z$ (with respect to $\star \Gamma_{\circ}=\mathbb{R}$ ), and gives the "rotating lines" foliation, whose restriction to $\mathbb{C}^{\times}$is given by the pairs of opposite rays $V=\arg z=s+k \pi, k=0,1$.

The metric

$$
g=\frac{d x^{2}+d y^{2}}{x^{2}+y^{2}}
$$

makes $\mathbb{C}^{\times}$a standard infinite cylinder of circumference $\mathcal{L}=\int_{S^{1}}|\omega|=\int_{S^{1}}-i \omega=2 \pi$, with (identity component 
of the) isometry group generated by $X, Y$. Restriction to a symmetric time interval $|t|<\mu / 2$ gives the finite cylinder $S^{1} \times(-\mu / 2, \mu / 2)$ and corresponding annulus $A_{r}=\{z: 1 / r<|z|<r\}, r=e^{\mu / 2}$, which provides the normal form in our discussion of foliated ring domains below. The modulus $\mu=\ln r^{2}$ of $A_{r}$ is related to the geometry of the cylinder by $\mu=2 \pi \mathcal{A} / \mathcal{L}^{2}=h$. The self-explanatory formula reflects the general characterization of ring domain moduli as solutions to extremal problems (the length-area method); a related extremal problem will play a role in the next section.

(b) In the second case,

$$
\begin{aligned}
\omega & =2 i \frac{d z}{z^{2}-1}=d \phi, \\
\phi & =i \log \frac{z-1}{z+1}
\end{aligned}
$$

and

$$
\begin{aligned}
\zeta\left(t, e^{i \theta}\right) & =\frac{\left(e^{i \theta}+1\right)+e^{-i t}\left(e^{i \theta}-1\right)}{\left(e^{i \theta}+1\right)-e^{-i t}\left(e^{i \theta}-1\right)} \\
& =\frac{\cos \frac{1}{2}(\theta-t)+i \sin \frac{1}{2}(\theta+t)}{\cos \frac{1}{2}(\theta+t)-i \sin \frac{1}{2}(\theta-t)} .
\end{aligned}
$$

The $2 \pi$-periodic geodesic $\Gamma_{t}$ foliates $\hat{\mathbb{C}} \backslash\{-1,1\}$ by circles through $z= \pm 1$, namely, the pairs of level sets $U(z)=-\arg \frac{z-1}{z+1}=t \mp \frac{\pi}{2}$; here the two signs $\mp$ correspond to the two circular arcs meeting at $\pm 1 ; \star \Gamma_{s}$ consists of the orthogonal circles $V(z)=\ln \left|\frac{z-1}{z+1}\right|=s$.

The points $\pm 1 \in S^{1}$ are pivot points of $\Gamma_{t}$ - stationary points at which the tangent line to $\Gamma_{t}$ rotates. In general, pivot points correspond to simple poles of $\omega$, and the residue of $\omega$ at such $z_{0}$ determines the constant rate of rotation of $\Gamma_{t}$ (as shown in [Calini and Langer 03]):

$$
\frac{d \alpha}{d t}=\frac{-i}{\operatorname{res}_{z_{0}} \omega} .
$$

Thus, the rotation rates at $z_{0}= \pm 1$ are

$$
\frac{d \alpha}{d t}=\mp 1
$$

Up to conjugation by a Möbius transformation, cases (a) and (b) are essentially the same, but with the roles of $\Gamma_{t}$ and $\star \Gamma_{s}$ reversed. In particular, $\hat{\mathbb{C}} \backslash\{-1,1\}$ is isometric to the cylinder of part (a). This is a good place to take note of a divergence between the foliated flat geometry and geodesic interpretations. On the one hand, there is no place for the points \pm 1 themselves in the cylindrical geometry $(\hat{\mathbb{C}} \backslash\{-1,1\}, g)$. On the other hand, these pivot points belong to the corresponding geodesic $\Gamma_{t}$, interpreted as a continuous variation of $S^{1}$ given by $\zeta$ or the Schwarz function

$$
S(t, z)=\frac{(z+1)+e^{2 i t}(z-1)}{(z+1)-e^{2 i t}(z-1)} .
$$

The same reasoning requires two values $U(z)=t \mp \frac{\pi}{2}$ above $(V=\arg z=s+k \pi$ in (a)), illustrating the fact that a given curve $\Gamma_{t_{0}}$ need not belong to a single trajectory of $p$.

(c) In the last case we have

$$
\begin{aligned}
\omega & =\frac{-d z}{(z-1)^{2}}=d \phi, \\
\phi & =\frac{1}{z-1}, \\
\zeta\left(t, e^{i \theta}\right) & =\frac{e^{i \theta}+t\left(e^{i \theta}-1\right)}{1+t\left(e^{i \theta}-1\right)},
\end{aligned}
$$

and $\Gamma_{t}, \star \Gamma_{s}$ foliate $\hat{\mathbb{C}} \backslash\{1\}$ by circles tangent to the line $x=1$ at $z=1$,

$$
U(z)=\Re\left(\frac{1}{z-1}\right)=\frac{x-1}{(x-1)^{2}+y^{2}}=t-\frac{1}{2},
$$

and the orthogonal circles

$$
V(z)=\Im\left(\frac{1}{z-1}\right)=\frac{-y}{(x-1)^{2}+y^{2}}=s .
$$

The map $\phi: \hat{\mathbb{C}} \backslash\{1\} \rightarrow \mathbb{C}$ defines an isometry between $(\hat{\mathbb{C}} \backslash\{1\}, g)$ and the Euclidean plane. The vanishing of the residue of $\omega$ at 1 implies that the Kasner invariant $\mathcal{K}$ associated with the pair of tangent curves $\Gamma_{\circ}$ and $\Gamma_{t}$ is constant in time $(\mathcal{K}=0$, actually); see [Calini and Langer 03]. Another exceptional feature of this case is the fact that the isometry group is three-dimensional, generated by $X, Y$ and the rotation field

$$
Z=\Re\left(-i(z-1) \partial_{z}\right)
$$

of course, rotations do not preserve the horizontal foliation. This phenomenon is explained in more general Lie-algebraic terms in Remark 10.4.

The following example provides a recipe for a large family of geodesics that are locally equivalent to the expanding circles $C_{e^{t}}$, and illustrates the computational value of some of the above quantities.

Example 4.2. (Poles of $\boldsymbol{\omega}$ as obstacles.) One may design "obstacle courses" for geodesics by placing $N$ pairs of 

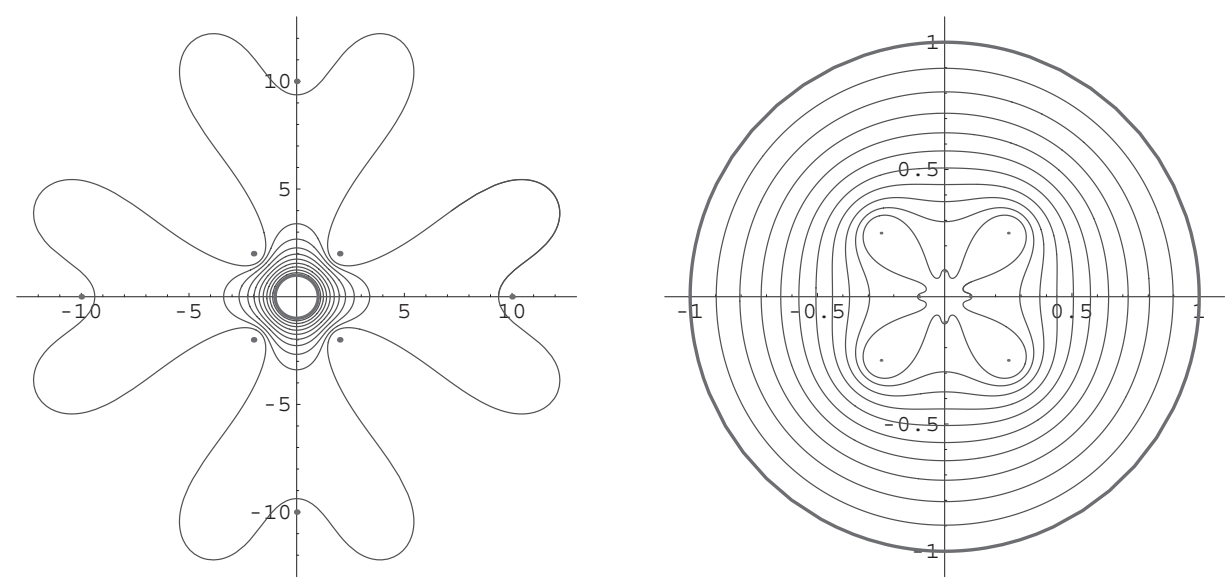

FIGURE 1. Poles of $\omega$ as obstacles for a geodesic $\Gamma_{t}$, shown for $t>0$, left, and for $t<0$, right.

poles $\alpha_{n}, 1 / \bar{\alpha}_{n}$ off the initial curve $S^{1}$. Consider

$$
g(z)=\frac{C z^{N}}{\prod_{n=1}^{N}\left(z-\alpha_{n}\right)\left(1-\bar{\alpha}_{n} z\right)}, \quad C \in \mathbb{R}^{\times},
$$

a general rational function that is real on $S^{1}$ and nonvanishing away from $0, \infty$. Define the differential $\omega=$ $g(z) d z / z$ and dual vector field $W=\frac{z}{g(z)} \partial z$. The flows of $X=\Re[W], Y=\Im[W]$ may be used to generate a figure consisting of curves $\Gamma_{j \Delta t},-J \leq j \leq J$, spaced by uniform time increment $\Delta t$; each curve is a trajectory of $X$, with initial condition determined by the conjugate flow $Y$, starting at a convenient point on $\Gamma_{\circ}=S^{1}$. Since $W$ is holomorphic on $\mathbb{C}^{\times}, \Gamma_{t}$ evolves smoothly-avoiding the zeros $\alpha_{n}, 1 / \bar{\alpha}_{n}$ of $W$-until $\Gamma_{t}$ reaches the singularities $0, \infty$. An example with $N=8$ is shown in Figure 1 (indicated in the figure are the "obstacles" $\pm 2 \pm 2 i, \pm 10, \pm 10 i$ and conjugate reciprocals).

Due to the singularities, the required computations are numerically sensitive. The following procedure yields good results. First, a residue calculation determines $C$ such that $\mathcal{L}=\int_{S^{1}}|\omega|=2 \pi$; this standardizes the parameter domain $[0,2 \pi]$ for the flow $Y$ (and makes $\Gamma_{t} \sim C_{e^{t}}$ ). Thus normalized, $\omega=d \phi$ is antidifferentiated to give a (multivalued) potential $\phi$. Using the real part $U=\Re(\phi)$, a potential difference $T=U(1)-U(0)=U(\infty)-U(1)$ is computed to determine the time it takes $\Gamma_{t}$ to run into $0, \infty$. To display a bounded but near maximal geodesic, one then makes choices to satisfy $J \Delta t=(1-\epsilon) T$, for small $\epsilon>0$. In the figure, $\epsilon=10^{-4}$; we note that the four "clover leaves" have scarcely begun to form until the last "instant." This is the reason $T$ must be computed precisely.

Applying contour-plot methods directly to the real potential $U$ might appear to be a simpler alternative to solving ODEs as above. We emphasize, however, that a given curve $\Gamma_{t_{0}}$ does not quite coincide with a $U$-level set; in the present example, the latter may have "unwanted components" (even as level sets were "too small" in Example 4.1).

Example 4.3. (String interactions.) Selecting $N$ pairs of simple poles $\alpha_{n}, 1 / \bar{\alpha}_{n}$ and corresponding real residues $\pm C_{n} \in \mathbb{R}^{\times}$, we consider the differential

$$
\omega=\frac{g(z) d z}{z}=\sum_{n=1}^{N} C_{n}\left(\frac{d z}{z-\alpha_{n}}-\frac{d z}{1-\bar{\alpha}_{n} z}\right) .
$$

As $t \rightarrow \pm \infty$, the asymptotic behavior of the corresponding flow $X=\Re(W)$ near these poles resembles that of Example 4.1(a) near 0, $\infty$; but $\omega$ also has $2 N-2$ zeros, disrupting smooth evolution of $\Gamma_{t}$ at intermediate times.

Here it is tempting to consider an alternative to the notion of geodesic as smoothly evolving Jordan curve $\Gamma_{t}$. Namely, $\omega=d \phi$ has single-valued real potential $U=\Re(\phi)$, whose level sets $U=t$ define a solution $\Gamma_{t}$ to our geodesic equation except at finitely many pointtimes $\left(z_{j}, t_{j}\right)$ where topological transitions occur. (To compare with the singular geodesics considered in [Calini and Langer 03], the topological transitions corresponded to cusps of $\Gamma_{t}$ due to branch points of $W$, and $\Gamma_{t}$ was seen to be represented nevertheless by a smooth solution to a quartic second-order PDE.) Such a foliation by level sets $U=t$ is essentially the setting of "string diagrams" as considered, for example, in [Krichever and Novikov 87], where an arbitrary compact Riemann surface is allowed in place of $\hat{\mathbb{C}}$.

Here the strings admit the following geometric description. Outside of a large enough compact subset $S \subset \hat{\mathbb{C}} \backslash\left\{\alpha_{1}, 1 / \bar{\alpha}_{1}, \ldots \alpha_{N}, 1 / \bar{\alpha}_{N}\right\}$, an individual "string" $s_{n}(t)$ looks like the standard cylinder of radius $\left|C_{n}\right|$. The 


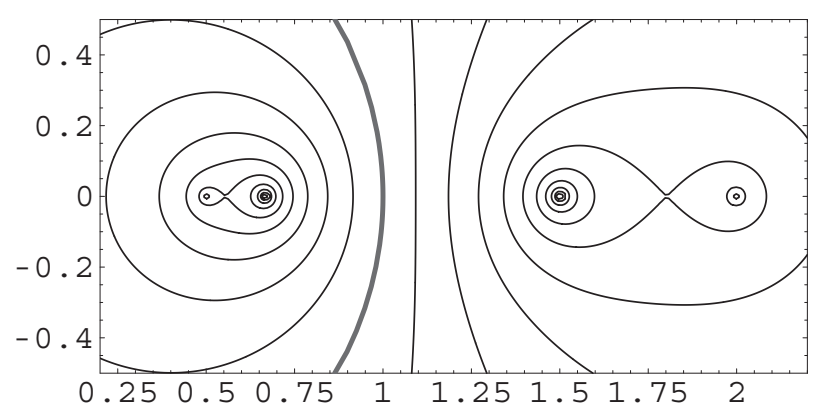

FIGURE 2. Two-string interaction.

"string interactions" are Euclidean surgeries among the cylinders taking place in $S$. In case $\left|\alpha_{n}\right|<1$ and $C_{n}>0$ for all $n$, we may assume that $\omega$ has been normalized so that $C_{1}+\cdots+C_{N}=1$ and consequently $S^{1}$ has length $\mathcal{L}=2 \pi$.

Figure 2 shows the interaction of two strings $s_{1}=$ $\left(\frac{1}{2}, 2\right)$ and $s_{2}=\left(\frac{2}{3}, \frac{3}{2}\right)$ with respective radii $C_{1}=\frac{5}{17}$ and $C_{2}=\frac{12}{17}$. Here one may recognize the pattern of Cassinian curves, symmetrized with respect to $S^{1}$ $(U=t=0)$; approximations of the classical family appear inside $(t<0)$ and outside $(t>0)$ the unit circle. To obtain satisfactory output using the Mathematica command ContourPlot, one need only choose a contour increment $\Delta U$ so that the lemniscate level sets $U= \pm U\left(z_{0}\right)$ are included; here, $z_{0}$ is one of the two zeros of $\omega, z_{0}=(33+\sqrt{305}) / 28 \approx 1.8$ (self-intersection point of the outside lemniscate). Finally, we note that by restricting time to $|t|<U\left(z_{0}\right)$, one may regard $\Gamma_{t}$ as a maximal geodesic in the smooth sense.

Figure 3 shows a variation on the above construction, in which $\omega=d \phi$ has a single pair of fifth-order poles $\alpha=\frac{3}{10}, 1 / \bar{\alpha}=\frac{10}{3}$, along with four pairs of simple zeros $\beta_{k}, 1 / \bar{\beta}_{k}$. The level sets $U=\Re(\phi)=t$ describe "selfinteractions of a single string" that are no longer asymptotically circular, but a "flower" with eight $(=2 \times 5-2)$ petals.

We wish to call attention to the more problematic zero-level set (highlighted), of which $S^{1}$ is a component. This figure illustrates but one of many possible ways the level-set interpretation $\Gamma_{t}=U^{-1}(t)$ could substantially complicate even a "short-time" or infinitesimal description of geodesics. Instead, the present paper emphasizes smoothly evolving Jordan curves $\Gamma_{t}$, allowing us to adopt simple heuristic interpretations of $\Lambda, T_{\circ} \Lambda$, and clearcut roles for the group $H=\operatorname{Diff}_{+}^{\omega}\left(S^{1}\right)$, the Witt algebra, etc. (In the future, we intend to explore interesting possibilities introduced by allowing variation fields, say, with poles on $\Gamma_{\circ}$, and further relations to [Krichever and

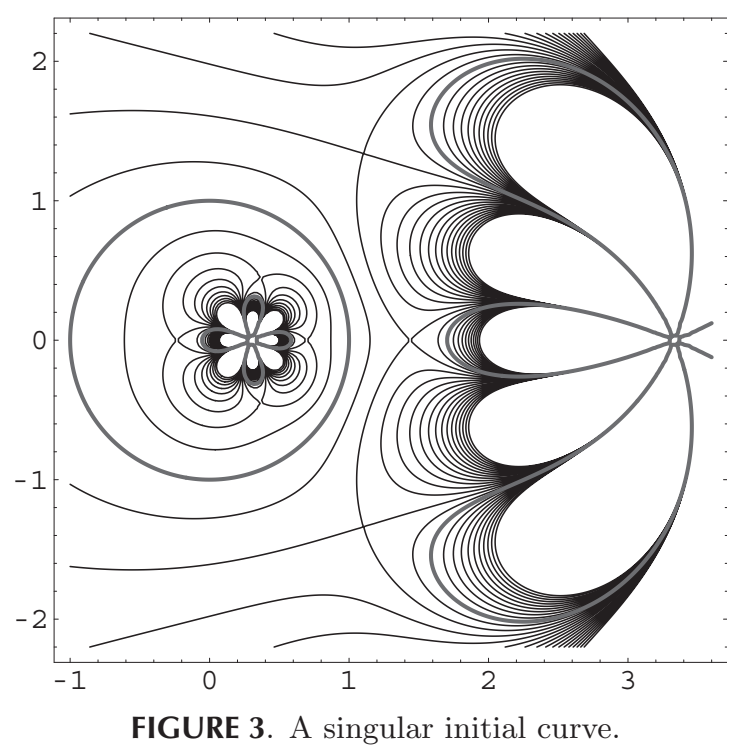

Novikov 87], in which a variety of Lie algebras are associated with curves in Riemann surfaces.)

Remark 4.4. With the previous paragraph in mind, all subsequent plots will display geodesics $\Gamma_{t}$ only up to a first encounter with singularities; thus, we have opted for clarity at the cost of sometimes losing information. (Arguably, the resulting figures are also aesthetically inferior to those just considered.) In the sense that a "typical" geodesic runs into a natural boundary anyway - this is certainly the appropriate viewpoint for the next sectionour blanket decision is further justified.

\section{MAXIMAL GEODESICS AND SINGULAR RING DOMAINS}

For $1<r<\infty$, let $A_{r}$ be the $S^{1}$-symmetric annulus

$$
A_{r}=\{z \in \mathbb{C}: 1 / r<|z|<r\} .
$$

As is well known, any member of the family $\mathcal{A}$ of ring domains $A \subset \hat{\mathbb{C}}$ (topological open annuli with both boundary components consisting of more than one point) is conformally equivalent to $A_{r}$ for exactly one value of $r$. The conformal class of $A$ is thus described by its symmetric radius $r=r(A)$, but more standardly by its modulus $\mu=2 \ln r$ (the height of the equivalent right circular cylinder of radius one). Given one such conformal equivalence $g: A_{r} \rightarrow A$, any other has the form $g\left(e^{i \theta_{0}} z\right)$ or $g\left(e^{i \theta_{0}} / z\right)$. If $A$ belongs to the family $\mathcal{A}_{\text {reg }} \subset \mathcal{A}$ of ring domains with boundary $\partial A$ consisting of two simple closed analytic curves, such $g$ extends conformally to $\partial A_{r}$. 
Any $A \in \mathcal{A}$ has a unique equator (or core curve), the closed analytic curve $\Gamma_{e} \subset A$ well defined by $\Gamma_{e}=g\left(S^{1}\right)$. There is an antiholomorphic involution of $A$ that switches boundary components with fixed point set $\Gamma_{e}$; no other points may be fixed by such a map. The core curve is also the unique closed geodesic with respect to the complete hyperbolic metric on $A$ of curvature -1 [McMullen, p. 12]; in this metric, $\Gamma_{e}$ has length $1 / 2 \mu$. The "limiting case" $A_{\infty}=\hat{\mathbb{C}} \backslash\{0, \infty\}$ is not hyperbolic, but any circle $C_{R}=\{z:|z|=R\}$ may play the role of $\Gamma_{e}$.

The $S^{1}$-symmetric ring domains $\mathcal{A}^{\circ}=\{A: A=\beta(A)\}$ $(\beta(z)=1 / \bar{z})$ are precisely those with equator $\Gamma_{e}=S^{1}$. We may identify $\mathcal{A}_{\text {reg }}^{\circ}=\mathcal{A}_{\text {reg }} \cap \mathcal{A}^{\circ}$ with $\Lambda_{+} \subset \Lambda$, the simple closed analytic curves in $\hat{\mathbb{C}}$ exterior to $S^{1} ; \Gamma \in$ $\Lambda_{+}$determines $A_{\Gamma} \in \mathcal{A}_{\text {reg }}^{\circ}$ with boundary components $\Gamma$, $\beta(\Gamma) \in \Lambda_{-}$. We define the radius of $\Gamma \in \Lambda_{+}$by $r(\Gamma)=$ $r\left(A_{\Gamma}\right)$ and by $r(\Gamma)=1 / r\left(A_{\beta(\Gamma)}\right)$ for $\Gamma \in \Lambda_{-}$. (We note that $r$ is the usual radius for circles $C_{r}$, but it is not the conformal radius of $\Gamma$ defined in [Kirillov 95], which is based on conformal mapping of disks rather than ring domains.)

Over the time interval $-\mu / 2<t<\mu / 2$, our expanding-circles geodesic $C_{e^{t}}=\exp \left(t z \partial_{z}\right)$ (Example 4.1) foliates $A_{r}, r=e^{\mu / 2}$. A conformal image $A=g\left(A_{r}\right)$ is likewise foliated by $\Gamma_{t}=g\left(C_{e^{t}}\right)=\exp \left(t \lambda_{g *} z \partial_{z}\right)$ over the same time interval; the duration of $\Gamma_{t}$ is the modulus of $A$. Conjugation of $e^{-2 t} z$ by $g$ gives the corresponding Schwarz function relative to the base curve $\Gamma_{\circ}=\Gamma_{e}=g\left(S^{1}\right)$.

In the symmetric case $A=g\left(A_{r}\right) \in \mathcal{A}^{\circ}, g$ preserves $\Gamma_{\circ}=S^{1}$, and may therefore be regarded as the unique continuation of an analytic diffeomorphism $h=\left.g\right|_{S^{1}} \in$ $H=\operatorname{Diff}_{+}^{\omega}\left(S^{1}\right)$. From now on we may write $h$ in place of $g$. (That $h$ is orientation-preserving on $S^{1}$ corresponds to $h\left(C_{e^{t}}\right)$ moving "outward.") We thus have a local action of $H$ on $\Lambda_{ \pm}$(or on $\mathcal{A}^{\circ}$ ), which is transitive on each level set $\Lambda_{r}$ of the radius function $r: \Lambda_{ \pm} \mapsto(0,1) \cup(0, \infty)$ by the conformal mapping facts quoted above. One also knows that the isotropy subgroup of a circle $C_{r}$ consists of rotations about the origin; but one cannot identify $\Lambda_{r}$ with $H / S^{1}$, since the action is only local.

On the other hand, the corresponding infinitesimal action is globally defined in the sense that for any $h \in H$ and for any analytic vector field $W=w(z) \partial_{z} \in T_{\Gamma_{\mathrm{o}}} \Lambda$ along $S^{1}$, we can compute $\lambda_{h *} W$ as in (3-3). In particular, we may associate with any coset $h S^{1} \subset H$ a maximal geodesic $\Gamma_{t}=\exp \left(t \lambda_{h *} z \partial_{z}\right),-m / 2<t<m / 2$, foliating a symmetric annular domain $A(h)=h\left(A_{R}\right)$, $R=R(h)=e^{m / 2}$. Here $A_{R}$ is maximal with respect to conformal continuations of the form $h: A_{r} \rightarrow \hat{\mathbb{C}}$. We call $R=R(h)$ the injectivity radius of $h \in H$. On $A(h)$, $W=\lambda_{h *} z \partial_{z}$ is a nonvanishing holomorphic vector field whose real and imaginary parts are, respectively, "normal" and "tangent" to the boundary $\partial A(h)$ (as well as to $\left.S^{1}\right)$. Namely, $\Im(W)$ generates the one-parameter group of automorphisms of $A(h)$ (which fixes the equator $S^{1}$ ), and the commuting fields $\Re(W), \Im(W)$ are coordinate fields whose flows may be used to recover the conformal map $h: A_{R} \rightarrow A(h)$.

The ring domain $A(h)$ is necessarily singular:

$$
A(h) \in \mathcal{A}_{\text {sing }}^{\circ}=\mathcal{A}^{\circ} \backslash \mathcal{A}_{\text {reg }}^{\circ}
$$

In the simplest case,

$$
h(z)=\frac{z-\alpha}{1-\bar{\alpha} z},
$$

an automorphism of the unit disk $A(h)$ misses just two points of $\hat{\mathbb{C}}$. The corresponding maximal geodesics $\Gamma_{t}$ are easily shown to be the only ones with $R=\infty$. For a finite geodesic $\Gamma_{t}, h$ might still continue analyticallybut nonunivalently - to the boundary $C_{R}$, with points of self-tangency $h\left(R^{i \theta_{1}}\right)=h\left(R^{i \theta_{2}}\right)$ or nonregular points $h\left(R^{i \theta_{0}}\right)$ with $h^{\prime}\left(R^{i \theta_{0}}\right)=0$ at the ends

$$
\Gamma_{m / 2}=h\left(C_{R}\right), \quad \Gamma_{-m / 2}=h\left(C_{1 / R}\right) \notin \Lambda_{ \pm} .
$$

(Such behavior is illustrated in the next example.) But in general, $h$ does not extend analytically to (the even part of) $C_{R}$, and $\Gamma_{ \pm m / 2}$ may exhibit all possible complexity of a natural boundary. (All the more curious is the implicit identification $\mathcal{A}_{\text {sing }}^{\circ} \simeq H / S^{1}$.)

Example 5.1. (Extremal ring domain.) We construct a geodesic from $\exp \left(t z \partial_{z}\right)$ as above by realizing the symmetrized Teichmüller domain

$$
T_{\rho}=\hat{\mathbb{C}} \backslash\{[0,1 / \rho] \cup[\rho, \infty]\}, \quad \rho>1,
$$

as the conformal image of a standard annulus $A_{R}$. The singular ring domain $T_{\rho}$ is the solution to an extremal problem to be utilized below. The required conformal map $h$ is given in terms of the Jacobi elliptic sine function $\operatorname{sn}(z, p)$ with modulus $p, 0<p<1$ (or parameter $m=$ $\left.p^{2}\right)$. We recall that $\operatorname{sn}(z, p)$ is an odd, doubly periodic meromorphic function on $\mathbb{C} ; \operatorname{sn}(z, p)$ has periods $4 K$ and $2 i K^{\prime}$, where

$$
K=K(p)=\int_{0}^{1} d x / \sqrt{\left(1-x^{2}\right)\left(1-p^{2} x^{2}\right)}
$$

is the complete elliptic integral of the first kind 


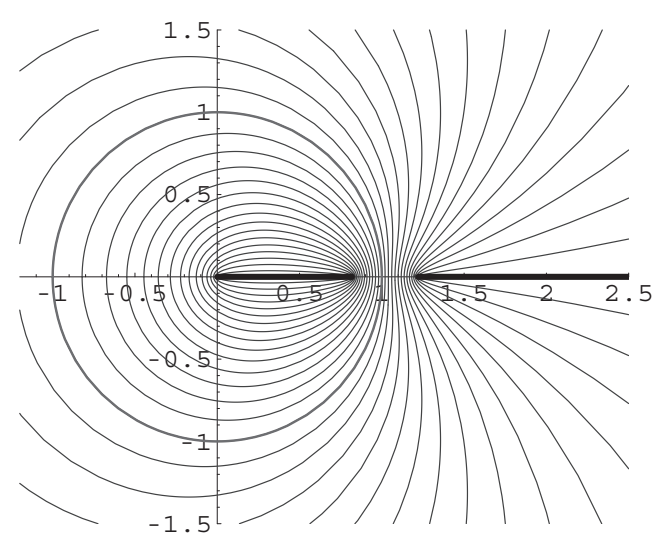

FIGURE 4. Maximal geodesic $\Gamma_{t}=\exp (t W)$ filling the extremal ring domain $T_{\rho}=\hat{\mathbb{C}} \backslash\left\{\left[0, \frac{9}{11}\right] \cup\left[\frac{11}{9}, \infty\right]\right\}$.

and $K^{\prime}=K^{\prime}(p)=K\left(1-p^{2}\right)$ its complement; $\operatorname{sn}(z, p)$ has a simple zero at the origin, simple poles at $\pm i K^{\prime}$, critical points at $\pm K$, and is otherwise free of such points on the closure of

$$
\mathcal{R}=\left\{z=x+i y:-K<x<K,-K^{\prime}<y<K^{\prime}\right\} .
$$

Finally, $\operatorname{sn}(z, p)$ is univalent on $\mathcal{R}$ and maps the latter conformally onto the domain

$$
S_{p}=\hat{\mathbb{C}} \backslash\left\{\left[-\frac{1}{p},-1\right] \cup\left[1, \frac{1}{p}\right]\right\} .
$$

Now $h: A_{R} \rightarrow T_{\rho}$ is defined as follows. Letting $R=e^{\pi K / K^{\prime}}$, we first map $A_{R}$ onto $\mathcal{R}$ via $z \mapsto w=$ $\frac{K^{\prime}}{\pi} \log z$. Then $\mathcal{R}$ is mapped as above onto $S_{p}$ via $w \mapsto Z=\operatorname{sn}(w, p)$. Finally, letting $\rho=\frac{1+p}{1-p}, S_{p}$ is taken to $T_{\rho}$ by $Z \mapsto \zeta=\frac{1+p Z}{1-p Z}$.

The canonical parametrization of the resulting maximal geodesic $\Gamma_{t}$ is then given in terms of the elliptic modulus $p$ by

$$
\begin{gathered}
\zeta=h\left(e^{t+i \theta}\right)=\frac{1+p \operatorname{sn}\left(\frac{K^{\prime}}{\pi}(t+i \theta), p\right)}{1-p \operatorname{sn}\left(\frac{K^{\prime}}{\pi}(t+i \theta), p\right)}, \\
-\pi \leq \theta<\pi, \quad-\frac{m}{2}<t<\frac{m}{2} \\
m=\mu\left(T_{\rho}\right)=2 \ln R=2 \pi \frac{K(p)}{K^{\prime}(p)} .
\end{gathered}
$$

Here, $m$ is the modulus of the above ring domains $A_{R}, S_{p}, T_{\rho}$. Note that $h$ extends analytically but not conformally to the boundary of $A_{R}$; in fact, $h^{\prime}\left(e^{ \pm \mu / 2}\right)=$ $h^{\prime}\left(-e^{ \pm \mu / 2}\right)=0$ and $h\left(e^{ \pm \mu / 2+i \theta}\right)=h\left(e^{ \pm \mu / 2-i \theta}\right), 0<$ $\theta<\pi$. The maximal geodesic $\Gamma_{t}$ for $p=\frac{1}{10}$ is shown in Figure 4; $\Gamma_{t}$ fills the ring domain $T_{\rho}=\hat{\mathbb{C}} \backslash$ $\left\{\left[0, \frac{9}{11}\right] \cup\left[\frac{11}{9}, \infty\right]\right\}$, which has modulus $m \approx 2.68$.
One may check that $\Gamma_{t}=\exp (t W)$, where $W$ is holomorphically defined on $T_{\rho}$ by

$$
W=\lambda_{h *} z \partial_{z}=\frac{2 K^{\prime}}{(1+\rho) \pi} \sqrt{z(\rho-z)(\rho z-1)} \partial_{z} .
$$

In the equivalent expression

$$
W=-\frac{2 i K^{\prime}}{(1+\rho) \pi} \sqrt{\rho^{2}+1-2 \rho \cos \theta} \partial_{\theta},
$$

the positive square root is chosen for $\theta \in \mathbb{R}$, so $W$ is outward along $S^{1}$.

Remark 5.2. Were it not for our present focus on maximal geodesics foliating singular ring domains $A \subset \hat{\mathbb{C}}$, it would be natural to regard the above $\Gamma_{t}$ as half of a closed geodesic foliating the torus $T^{2}=T_{\rho}^{+} \cup T_{\rho}^{-}$obtained by gluing two copies of $T_{\rho}$ together. We note that

$$
p=\left(\frac{(1+\rho) \pi}{2 K^{\prime}}\right)^{2} \frac{d z^{2}}{z(\rho-z)(\rho z-1)}
$$

defines a nonorientable quadratic differential on $\hat{\mathbb{C}}$, which lifts to orientable $\hat{p}$ on $T^{2}$. The resulting geodesic on $T^{2}$ corresponds to concatenation of $\exp (t W)$ with the other branch $\exp (-t W)$, yielding a closed geodesic of period $2 m$.

Next we characterize the orbit

$$
H \cdot z \partial_{z}=\left\{W=\lambda_{h *} z \partial_{z}\right\}_{h \in H} \subset T_{\Gamma_{\circ}} \Lambda
$$

in terms of $W\left(e^{i \theta}\right)$. First, $H \cdot z \partial_{z}$ lies in the open cone $\mathcal{C}_{+} \subset T_{\Gamma_{\circ}} \Lambda$ of "outward fields"

$$
W=z \nu(z) \partial_{z}=-i \nu\left(e^{i \theta}\right) \partial_{\theta}
$$

where $\nu\left(e^{i \theta}\right)>0$ for $e^{i \theta} \in S^{1}$. The dual differential $\omega=d z / w(z)$ of any $W=w(z) \partial_{z} \in \mathcal{C}_{+}$is analytic along $S^{1}$ and gives a positive value for the length of $S^{1}$ :

$$
\mathcal{L}(\omega)=\int_{S^{1}}|\omega|=\int_{S^{1}}-i \omega=\int_{0}^{2 \pi} \frac{d \theta}{\nu\left(e^{i \theta}\right)} .
$$

In particular, if $\omega$ is dual to $W \in H \cdot z \partial_{z}$, then $\mathcal{L}(\omega)=$ $\mathcal{L}(d z / z)=2 \pi$. Conversely, if $W=w \partial_{z} \in \mathcal{C}_{+}$satisfies $\mathcal{L}(d z / w)=2 \pi$, then $W \in H \cdot z \partial_{z}$. (This special case of Theorem 10.2 may be verified directly, using the natural parameters $\phi=\int_{1}^{\zeta} \omega$ and $\phi_{0}=\int_{1}^{z} \frac{d z}{z}$ to construct the required conformal map $z \mapsto \zeta=h(z)$ on some annu$\operatorname{lus} A_{\epsilon}$.)

Regarding the length condition $\mathcal{L}(\omega)=2 \pi$ as a kind of normalization, we will henceforth let $\hat{W}=\hat{w}(z) \partial_{z}$ 
denote an element of $\mathcal{C}_{+}$satisfying $\mathcal{L}(d z / \hat{w})=2 \pi$ (i.e., $\hat{W} \in H \cdot z \partial_{z}$ ); other vectors in $\mathcal{C}_{ \pm}= \pm \mathcal{C}_{+}$may then be written $W=\tau \hat{W}, \pm \tau>0$. Further, such $\hat{W}$ determines a maximal geodesic $\exp (t \hat{W}), 2|t|<m=m(\hat{W})$. The maximal subset of $\mathcal{C}_{ \pm}$on which the exponential map at $\Gamma_{\circ}$ is defined (in the above nonsingular sense) is then given by

$$
U_{ \pm}=\left\{W=\tau \hat{W} \in \mathcal{C}_{ \pm}: 0<|\tau|<m / 2\right\} .
$$

Starting with $\hat{W}$ as above, one would like to obtain information about $m$ based on the locations of singularities of $\hat{W}$; note that these must lie outside of $A(h)$ and occur in reflected pairs $\alpha, 1 / \bar{\alpha} \in \hat{\mathbb{C}}$, by virtue of the fact that $\hat{W} \in T_{\Gamma_{\circ}} \Lambda$ satisfies $\hat{W}^{*}=-\hat{W}$. Thus, $\hat{W}$ has a symmetric singular set, $\mathcal{S}=1 / \overline{\mathcal{S}} \subset \hat{\mathbb{C}} \backslash h(A)$, consisting of those $\alpha \in \hat{\mathbb{C}}$ that do not lie in any connected neighborhood of $S^{1}$ to which $\hat{W}$ extends holomorphically and nonsingularly. The set $\mathcal{S}$ contains at least two points $\alpha, 1 / \bar{\alpha}$, which we may take to be $0, \infty \in \mathcal{S}$ by trivially modifying $\hat{W}$ by the automorphism $\varphi_{\alpha}(z)=(z-\alpha) /(1-\bar{\alpha} z)$. Henceforth, we will assume that this has been done, and write simply $\hat{W}$ in place of $\lambda_{\varphi_{\alpha} *} \hat{W}$. We will give an upper bound on $m$ in terms of the regularity radius of $\hat{W}$ :

$$
\rho=\rho(\hat{W})=\sup \left\{r: A_{r} \cap \mathcal{S}=\varnothing\right\} .
$$

(Of course, $\rho$ depends on $\alpha$; one may reasonably eliminate this dependence by taking the infimum over the original choices $\alpha \in \mathcal{S}(\hat{W})$.)

Proposition 5.3. exp : $U_{ \pm} \rightarrow \Lambda_{ \pm}$is bijective, and maps $\tau \hat{W} \in U_{ \pm}$to a curve $\Gamma=\exp (\tau \hat{W})$ of radius $r(\Gamma)=e^{\tau}$. If $\hat{W}$ has singularities other than $0, \infty$, then $\hat{W}$ determines a finite maximal geodesic $\exp (t \hat{W}), 2|t|<m(\hat{W})$, which foliates a singular ring domain $A \in \mathcal{A}_{\text {sing. The }}$ duration $m(\hat{W})=\mu(A)$ is bounded above in terms of the regularity radius $\rho=\rho(\hat{W})$ by

$$
m \leq 2 \ln R=\frac{2 \pi K(p)}{K^{\prime}(p)}, \quad p=\frac{\rho-1}{\rho+1} .
$$

The bound is sharp, and is realized by the Teichmüller extremal domain

$$
T_{\rho}=\hat{\mathbb{C}} \backslash\{[0,1 / \rho] \cup[\rho, \infty]\}=h\left(A_{R}\right),
$$

with $h$ explicitly constructed via $\operatorname{sn}(z, p)$, the elliptic sine function of modulus $p$. It follows that $R(A)<4 \rho$, with 4 the best possible constant.

Proof: Surjectivity of exp follows from conformal mapping as above. Namely, for $\Gamma \in \Lambda_{+}$, there exists $h: A_{r} \rightarrow$
$A_{\Gamma}$ conformal up to the boundary $\partial A_{r}$, so $R(h)>r$. Letting $\hat{W}=\lambda_{h *} z \partial_{z}$ and $\tau=\ln r$, it follows that $\tau \hat{W} \in U_{+}$. Then

$$
\begin{aligned}
\exp (\tau \hat{W}) & =\exp \left(\lambda_{h *}\left(\tau z \partial_{z}\right)\right)=\lambda_{h}\left(\exp \left(\tau z \partial_{z}\right)\right) \\
& =h\left(C_{r}\right)=\Gamma
\end{aligned}
$$

and $r(\Gamma)=r\left(C_{r}\right)=r$. Also,

$$
\exp (-\tau \hat{W})=\beta(\Gamma)
$$

so any $\Gamma \in \Lambda_{ \pm}$lies in the image of exp.

To prove injectivity of exp, suppose

$$
\exp (t \hat{W})=\exp (s \hat{V})=\Gamma,
$$

for some $t \hat{W}, s \hat{V} \in U_{ \pm}$. Then $s=\mu\left(A_{\Gamma}\right) / 2=t$, and $\hat{W}, \hat{V}$ are nonvanishing holomorphic vector fields on $A_{\Gamma}$, with imaginary parts generating one-parameter groups of automorphisms of $A_{\Gamma}$. Since $\operatorname{Aut}\left(A_{\Gamma}\right)$ is one-dimensional, $\hat{W}$ and $\hat{V}$ must therefore be proportional: $\hat{W}=c \hat{V}$, in which only $c=1$ is consistent with $\hat{W}, \hat{V}$ both being "normalized."

Finally, we show how to estimate $\mu$. By rotation about the origin, we may assume that the singular set of $\hat{W}$ includes the four points $\rho, 1 / \rho, 0, \infty \in \mathcal{S}(\hat{W})$. Like the symmetrized Teichmüller domain $T_{\rho}$, the ring domain $A(h)$ lies in the complement of these four points and separates $\{0,1 / \rho\}$ from $\{\rho, \infty\}$. But the former is known to be extremal; that is, $T_{\rho}$ has the largest modulus among ring domains satisfying this description. Up to Möbius equivalence, this is a special case of Teichmüller's Module Theorem; see [Lehto and Virtanen 73, Theorem 1.1, p. 55], where Teichmüller domains are defined by removing segments $\left[-r_{1}, 0\right]$ and $\left[r_{2}, \infty\right]$ and need to be $S^{1}$-symmetrized for our purposes. Thus, the given bound on $\mu$ follows from the computations in Example 5.1, and sharpness of the bound follows as well.

The function

$$
f(p)=\frac{R\left(T_{\rho}\right)}{\rho}=\frac{1-p}{1+p} e^{\pi K(p) / K^{\prime}(p)}
$$

increases monotonically from 1 to a limiting value of 4 for $p$ in the interval $[0,1)$. The derivative of $f(p)$ is easily computed (using standard formulas for $\frac{d K}{d p}, \frac{d K^{\prime}}{d p}$ and the Legendre relation):

$$
f^{\prime}(p)=\frac{f(p)}{2 p\left(1-p^{2}\right) K^{\prime 2}}\left(\pi^{2}-4 p K^{\prime 2}\right) .
$$

Monotonicity thus reduces to the elementary estimate $p K^{\prime 2}<\pi^{2} / 4$. The upper value $\lim _{p \rightarrow 1} f(p)=4$ follows easily from [Byrd and Friedman 54, equation 112.04], where one may find also the other required facts about elliptic integrals. 

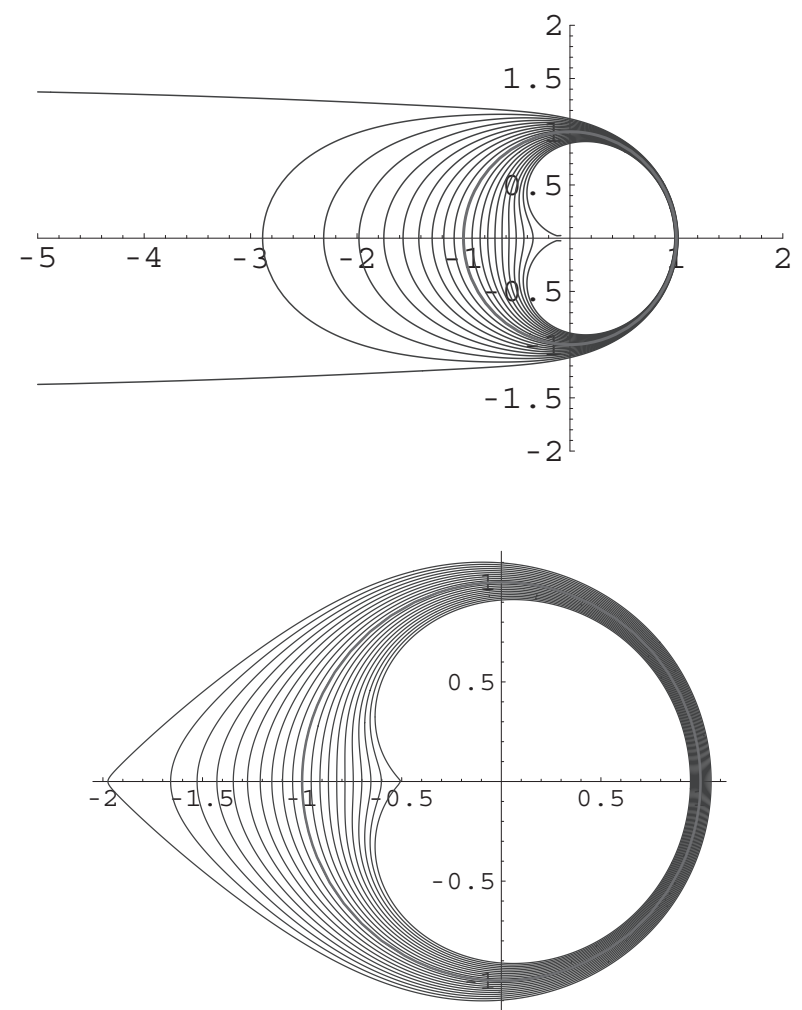

FIGURE 5. Maximal geodesics filling nonextremal singular ring domains.

It is easy to see that no corresponding lower bound on the ratio $R / \rho=e^{\frac{m}{2}} / \rho$ exists, as illustrated in the first part of the following example:

\section{Example 5.4. (Nonextremal singular ring domains.)}

(a) Consider the differential

$$
\omega=\frac{i}{a} e^{2 \cos \theta} d \theta=\frac{e^{z+1 / z} d z}{a z}
$$

where $a=\frac{1}{2 \pi} \int_{0}^{2 \pi} e^{2 \cos \theta} d \theta$. The dual "outward" vector field $\hat{W}$ along $S^{1}$ lies in the orbit $H \cdot z \partial_{z}$, since it satisfies the normalization $\mathcal{L}(\omega)=2 \pi$; the geodesics $\exp \left(t z \partial_{z}\right)$ and $\exp (t \hat{W})$ are thus locally equivalent. Since $\hat{W}$ has only the two essential singularities at $0, \infty$, it has infinite nonsingularity radius $\rho(\hat{W})=\infty$. Unlike $\exp \left(t z \partial_{z}\right)$, however, $\exp (t \hat{W})$ runs into $\infty, 0$ in finite time $t= \pm m(\hat{W}) / 2$ as it fills in a singular ring domain of modulus $m(\hat{W}) \approx 0.1$ and symmetric radius $R=e^{\frac{m}{2}} \approx 1.05$. The latter domain is shown in Figure 5 (top).

(b) Intermediate (and in some sense more typical) examples are provided by the family of differentials

$$
\begin{aligned}
\omega_{r} & =i d \theta+\frac{2 i r}{r^{2}+1} \cos \theta d \theta \\
& =\frac{d z}{z}+\frac{r}{r^{2}+1}\left(z+z^{-1}\right) \frac{d z}{z}, \quad 1<r<\infty .
\end{aligned}
$$

(One may regard $\omega_{r}$ either as a deformation of the standard differential $\omega_{\infty}=d z / z$, or as a "truncated" version of part (a) of this example.) The family $\omega_{r}$ has zeros at $-r$ and $-1 / r$ and second-order poles at $0, \infty$, so $\rho\left(\hat{W}_{r}\right)=r$. The maximal geodesic $\Gamma_{t}=\exp \left(t \hat{W}_{r}\right)$ foliates a singular ring domain $A$ of modulus

$$
\mu(A)=2 \Re(\phi(-r))=2\left(\ln r+\frac{1-r^{2}}{1+r^{2}}\right),
$$

where

$$
\phi(z)=\int_{1}^{z} \omega_{r}=\log z+\frac{r}{r^{2}+1}\left(z-z^{-1}\right) .
$$

The ratio of $\mu(A)$ to the upper bound $2 \pi K(p) / K^{\prime}(p)$, $p=(r-1) /(r+1)$, is small, for small $r$, but approaches 1 as $r \rightarrow \infty$.

The case $r=2$ is shown in Figure 5 (bottom); in this case $\mu(A) \approx 0.19$, while $2 \pi K / K^{\prime} \approx 4$. Again, the geodesic $\Gamma_{t}$ runs into singularities (at $-2,-\frac{1}{2}$ ) long before it has a chance to fill up much of the plane. In fact, in the right half-plane, $A$ nearly coincides with the round annulus of modulus $\mu=0.19$. As in (a), the much larger left half of the annulus has little influence on $\mu$. This reflects a basic principle of degenerating sequences of ring domains: if the boundary components approach each other 
while diameters of components themselves are bounded away from zero, the modulus tends to zero. (The quantitative expression of this principle is stated in terms of the spherical metric on $\mathbb{C}$ [Lehto and Virtanen 73, Lemma 6.2, p. 34].

Remark 5.5. In the remainder of the paper we consider the exponential map exp $=\exp _{\circ}$ at $S^{1}$ for tangent fields $W=w(z) \partial_{z}$ that may have zeros along $S^{1}$. As above, $\exp (W)$ is defined for those $W \in T_{0} \Lambda$ for which the geodesic equation has a solution $t \mapsto \Gamma_{t}=\exp (t W) \in \Lambda$ for $t \in[0,1]$. The zeros do not interfere with exponentiation per se, but the resulting geodesics have stationary points on $S^{1}$ and fail to sweep out planar domains; consider, for example, $\exp \left(t W_{+}\right), \exp \left(t W_{0}\right)$ as in Example 4.1. Therefore one cannot directly apply standard conformal mapping theory to obtain global results as above. In particular, in place of the easily answered question of surjectivity of the restricted mapping exp : $U_{ \pm} \rightarrow \Lambda_{ \pm}$, one confronts already the following hard problem

Problem 5.6. Describe the domain $U \subset T_{0} \Lambda$ and range $V \subset \Lambda$ of exp.

The local version of this problem obtained by restricting to a neighborhood of a stationary point on $S^{1}$ is essentially the question of when two pairs of intersecting curves (curvilinear angles or horn angles in the case of curves meeting tangentially) are conformally equivalent; we refer the reader to [Davis 74] and references therein for an indication of the analytical subtleties inherent in this and related problems.

\section{THE INFINITESIMAL SYMMETRIC SPACE $T_{\circ} \Lambda$}

To study the infinitesimal geometry of the space $\Lambda$ of unparametrized analytic curves, we may fix a convenient base curve $\Gamma_{\circ}$ and identify nearby curves $\Gamma \in \Lambda$ with elements of $\mathcal{S}_{0}$, the space of Schwarz functions relative to $\Gamma_{\circ}$. Since we wish to make use of the Lie algebra $\mathfrak{g}$ of analytic vector fields defined along the circle, the natural choice is $\Gamma_{\circ}=S^{1}$ (even though $\Gamma_{\circ}=\hat{\mathbb{R}}$ is simpler for certain computations).

We consider $G$ consisting of analytic diffeomorphisms defined near $\Gamma_{\circ}=S^{1}$ and the group $H=\operatorname{Diff}_{+}^{\omega}\left(S^{1}\right) \subset G$ of analytic diffeomorphisms preserving $S^{1}$ (and its orientation). We write $T_{\text {Id }} G \cong \mathfrak{g} \cong \mathfrak{h} \oplus i \mathfrak{h}$, expressing elements in either notation $A=a(\theta) \partial_{\theta}$ or $A=i z \mathbf{a}(z) \partial_{z}$. The case $A \in \mathfrak{h}$ corresponds to $\mathbf{a}(z)$ being real on $S^{1}$ (i.e., $a(\theta)=\mathbf{a}\left(e^{i \theta}\right)$ is real for $\theta \in \mathbb{R}$ ), and $A$ may be regarded as a real analytic tangent field along $S^{1}$. Likewise, $-i A=-i a(\theta) \partial_{\theta}=z \mathbf{a}(z) \partial_{z}$ denotes an element of $i \mathfrak{h}$, with the same reality condition on $\mathbf{a}(z)$, and may be interpreted as a normal vector field along $S^{1}$. In view of earlier discussion, we thus make the identifications $T_{\mathrm{o}} \Lambda \cong T_{\mathrm{Id}} \mathcal{S}_{\circ} \cong i \mathfrak{h}$.

Then $A \in \mathfrak{g}$ generates a local flow $e^{t A}$ defined near $S^{1}$, and the formula

$\operatorname{Ad}_{g} A=\left.\frac{d}{d t} g \circ e^{t A} \circ g^{-1}\right|_{t=0}=g^{\prime} \circ g^{-1} A \circ g^{-1}=\left.g^{\prime} A\right|_{g^{-1}}$

defines the adjoint action Ad $: G \times \mathfrak{g} \rightarrow \mathfrak{g}$. The operator commutator of $A=i z \mathbf{a}(z) \partial_{z}, B=i z \mathbf{b}(z) \partial_{z} \in \mathfrak{g}$ is

$$
[A, B]=-z^{2}\left(\mathbf{a b}_{z}-\mathbf{a}_{z} \mathbf{b}\right) \partial_{z}=i^{2} z^{2} \mathbf{a}^{2}(\mathbf{b} / \mathbf{a})_{z} \partial_{z}=i z \mathbf{c} \partial_{z} .
$$

If $\mathbf{a}, \mathbf{b}$ are real on $S^{1}$, then so is $\mathbf{c}$, as is transparent in the other notation

$$
[A, B]=\left[a \partial_{\theta}, b \partial_{\theta}\right]=\left(a b^{\prime}-a^{\prime} b\right) \partial_{\theta}=a^{2}(b / a)^{\prime} \partial_{\theta}=c \partial_{\theta} .
$$

As usual, ad $: \mathfrak{g} \times \mathfrak{g} \rightarrow \mathfrak{g}$, defined as the differential of $g \mapsto \operatorname{Ad}_{g} A$ at $g=\mathrm{Id}$, is computed in terms of the commutator:

$$
\operatorname{ad}_{B} A=\left.\frac{d}{d u} \operatorname{Ad}_{e^{u B}} A\right|_{u=0}=[A, B] .
$$

(The above notation fits the usual identification of the Lie algebra of a diffeomorphism group $G$ with the rightinvariant vector fields on $G$ [Milnor 84] explaining the sign difference with the most-standard matrix group convention $\operatorname{ad}_{A} B=[A, B]$ based on left-invariant vector fields.)

Either (6-2) or (6-3) yields the Witt algebra bracket relations

$$
\begin{aligned}
d_{n} & =-z^{n+1} \partial_{z}=i e^{i n \theta} \partial_{\theta}, \\
{\left[d_{m}, d_{n}\right] } & =(m-n) d_{m+n}, \quad m, n \in \mathbb{Z} .
\end{aligned}
$$

We will also use the basis

$$
c_{0}=\partial_{\theta}, \quad c_{n}=\cos (n \theta) \partial_{\theta}, \quad s_{n}=\sin (n \theta) \partial_{\theta},
$$

$n=1,2,3, \ldots$, for the real algebra $\mathfrak{h}$, though the bracket relations for $c_{0}=-i d_{0}, c_{n}=-\frac{i}{2}\left(d_{n}+d_{-n}\right)$, and $s_{n}=$ $-\frac{1}{2}\left(d_{n}-d_{-n}\right)$ are not as convenient.

We return now to the geometry of $\Lambda$, and the heuristic $\Lambda \cong G / H$ (unparametrized curves are parametrized curves modulo reparametrizations). The corresponding infinitesimal homogeneous space (or infinitesimal Klein geometry as in [Sharpe 97]) is a geometry on the space 
$T_{\mathrm{\circ}} \Lambda$ with symmetries defined by the isotropy representation $\mathrm{Ad}^{\Lambda}: H \rightarrow \mathrm{GL}\left(T_{\circ} \Lambda\right)$. In the reductive case $\mathfrak{g} \cong$ $\mathfrak{h} \oplus \mathfrak{m}$, with $\operatorname{Ad}_{H}(\mathfrak{m}) \subset \mathfrak{m}$, one may identify $\operatorname{Ad}^{\Lambda}$ with the "second factor" of the restriction to $H$ of $\mathrm{Ad}: G \times \mathfrak{g} \rightarrow \mathfrak{g}$ [Guest 97, p. 16]. The present case is especially simple, with $\mathfrak{m}=i \mathfrak{h}$ and $\mathrm{Ad}^{\Lambda}$ related to $\left.\mathrm{Ad}\right|_{H}$ by complex linearity.

Comparison of (3-3) and (6-1) verifies that we have simply recovered the action previously considered,

$$
\operatorname{Ad}^{\Lambda}=\lambda_{*}: H \times T_{\circ} \Lambda \rightarrow T_{\circ} \Lambda
$$

placing the latter in a homogeneous-space context. (Here we have restricted $\lambda_{*}$ to $H$, incidentally, resulting in an actual group action on $T_{\circ} \Lambda$ by the Fréchet Lie group $H$, etc., but we will make no use of infinite-dimensional analysis here.) Finally, we mention that $\mathfrak{g} \cong \mathfrak{h} \oplus i \mathfrak{h}$ is the decomposition by \pm 1 -eigenspaces of the operation

$$
W=\mathbf{w}(z) \partial_{z} \mapsto W^{*}=-z^{2} \mathbf{w}^{*}(z) \partial_{z}
$$

(dual to the operation on differentials $\omega \mapsto \omega^{*}$ discussed in Section 9). The involution * on $\mathfrak{g}$ yields the standard construction of the Lie triple system $[X, Y, Z]=$ $[[X, Y], Z]$ on $\mathfrak{g}_{-}=i \mathfrak{h}[\operatorname{Loos} 69]$.

Equation (6-5) implies an important fact about the geometry of $T_{\circ} \Lambda$. Consider a bilinear form $\langle\rangle:, \mathfrak{g} \times \mathfrak{g} \rightarrow$ $\mathbb{C}$ that is invariant with respect to the adjoint action, that is, satisfying $\left\langle\operatorname{Ad}_{g} \alpha, \operatorname{Ad}_{g} \beta\right\rangle=\langle\alpha, \beta\rangle$ for all $\alpha, \beta \in \mathfrak{g}$, $g \in G$. Differentiation with respect to $g$ at $g=\mathrm{Id}$ gives the ad-invariance condition $\left\langle\operatorname{ad}_{\gamma} \alpha, \beta\right\rangle+\left\langle\alpha, \operatorname{ad}_{\gamma} \beta\right\rangle=0$, for all $\alpha, \beta, \gamma \in \mathfrak{g}$. Expressed in the most symmetrical form, the condition is

$$
\langle[\alpha, \beta], \gamma\rangle=\langle\alpha,[\beta, \gamma]\rangle=\langle\beta,[\gamma, \alpha]\rangle, \quad \alpha, \beta, \gamma \in \mathfrak{g}
$$

Take now $\alpha=d_{n}, \beta=d_{m}, \gamma=d_{-(n+m)}$, to get

$$
\begin{aligned}
(n-m)\left\langle d_{n+m}, d_{-(n+m)}\right\rangle & =(2 m+n)\left\langle d_{n}, d_{-n}\right\rangle \\
& =-(2 n+m)\left\langle d_{m}, d_{-m}\right\rangle .
\end{aligned}
$$

If $n=m \neq 0$, then $\left\langle d_{n}, d_{-n}\right\rangle=0$. On the other hand, the choice $n=-m$ leads to the equation $2 n\left\langle d_{0}, d_{0}\right\rangle=$ $-n\left\langle d_{n}, d_{-n}\right\rangle=0$, implying $\left\langle d_{0}, d_{0}\right\rangle=0$ (using $\left.n \neq 0\right)$. Thus, as is well known, there does not exist a nondegenerate bilinear form on $\mathfrak{g}$ that is invariant with respect to the adjoint action. (The related Remark 10.3 highlights simple differences between adjoint and coadjoint actions.) This conclusion is equivalent to corresponding statements about the restricted actions Ad : $H \times \mathfrak{h} \rightarrow \mathfrak{h}$ and $\lambda_{*}: H \times T_{\circ} \Lambda \rightarrow T_{\circ} \Lambda$, by virtue of complex extension.
Proposition 6.1. There does not exist a (pseudoRiemannian) metric on $\Lambda$ with respect to which the canonical left action $\lambda$ is isometric; in fact, no nonsingular bilinear form on $T_{\circ} \Lambda$ is preserved by $\lambda_{*}$.

Since $\lambda$ preserves the symmetric-space multiplication defining the geometry of $\Lambda$ - the covariant derivative, curvature, geodesics, etc.- the implication of the proposition is that $\Lambda$ is not a metric geometry. In this sense, the symmetric subspace of circles $\Lambda^{3}$ considered in [Calini and Langer 03] has more rigid structure. Viewed intrinsically, $\left(\Lambda^{3}, \cdot\right)$ happens to be a semisimple symmetric space [Loos 69, Chapter 4]; its Lorentzian metric and group of displacements $\operatorname{Isom}_{0}\left(\Lambda^{3}, \rho\right) \simeq \operatorname{SL}(2, \mathbb{C})$ may be recovered via the Ricci form $\rho(X, Y)=\operatorname{tr}(Z \mapsto R(Y, Z) X)$ and Lie triple system of $\left(\Lambda^{3}, \cdot\right)$, respectively. (What other pseudo-Riemannian geometries - not just symmetric subspaces - are naturally embedded in $\Lambda$ via our constructions is one of the general issues raised in [Calini and Langer 03] that we intend to address elsewhere.)

Another relevant feature of the Witt algebra is the fact that for each integer $n>1$, there is an isomorphism $\Phi_{n}$ : $\mathfrak{g} \rightarrow \mathfrak{g}_{n}$ mapping $\mathfrak{g}=\left\langle d_{j}\right\rangle$ onto a subalgebra $\mathfrak{g}_{n}=\left\langle D_{j}\right\rangle$, namely

$$
d_{j} \mapsto D_{j}=\Phi_{n}\left(d_{j}\right)=\frac{1}{n} d_{n j}
$$

The geometric significance of this fact is explained by the following heuristic argument. Letting $\varphi(z)=z^{1 / n}$, $\varphi^{-1}(z)=z^{n}$, and allowing ourselves to make the exponent cancellation $\left(z^{n}\right)^{1 / n}=z$, we compute

$$
\begin{aligned}
\operatorname{Ad}_{\varphi} d_{j} & =-\operatorname{Ad}_{\varphi} z^{j+1} \partial_{z}=-\frac{1}{n}\left(z^{n}\right)^{(1-n) / n}\left(z^{n}\right)^{j+1} \partial_{z} \\
& =-\frac{1}{n} z^{1+n j} \partial_{z}=\frac{1}{n} d_{n j} .
\end{aligned}
$$

In other words, the above isomorphism may be interpreted as the induced map $\Phi_{n}=\lambda_{\varphi *}=\operatorname{Ad}_{\varphi}$ associated with the conformal equivalence between $\mathbb{C}^{\times}$and its $n$-fold cover. While the global interpretation of this argument represents a basic challenge in the theory of $\Lambda$, its concrete significance is very simple: as the following example illustrates, $\varphi$ turns "old" geodesics into "new" ones.

Example 6.2. (Sinusoidal variations.) The geodesic $\exp (t W)$ may be computed explicitly if $i W$ is one of the Witt algebra basis elements $c_{n}, s_{n}$. It suffices to consider 


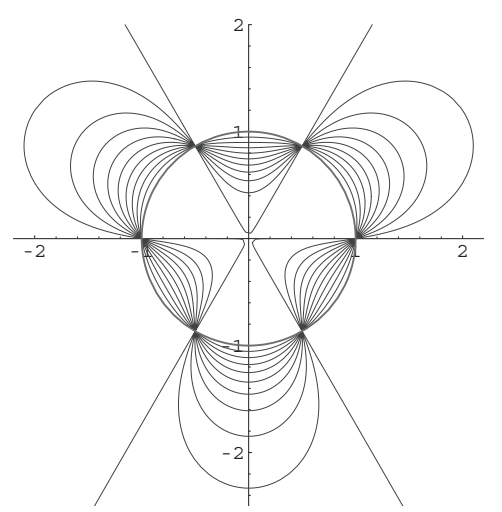

FIGURE 6. Geodesic with six pivot points.

the following expressions:

$$
\begin{aligned}
W_{n} & =\frac{1}{i n} s_{n}=\frac{1}{i n} \sin n \theta \partial_{\theta}=\frac{1}{2 i n} z\left(z^{n}-z^{-n}\right) \partial_{z} \\
\omega_{n} & =\frac{2 i n z^{n-1} d z}{\left(z^{n}-1\right)\left(z^{n}+1\right)}=\frac{i n z^{n-1} d z}{z^{n}-1}-\frac{i n z^{n-1} d z}{z^{n}+1} \\
\phi_{n} & =i \log \frac{z^{n}-1}{z^{n}+1} \\
U & =-\arg \frac{z^{n}-1}{z^{n}+1}=t-\frac{\pi}{2} \\
\zeta_{n}^{n} & =\frac{\left(e^{i n \theta}+1\right)+e^{-i t}\left(e^{i n \theta}-1\right)}{\left(e^{i n \theta}+1\right)-e^{-i t}\left(e^{i n \theta}-1\right)} \\
& =\frac{\cos \frac{1}{2}(n \theta-t)+i \sin \frac{1}{2}(n \theta+t)}{\cos \frac{1}{2}(n \theta+t)-i \sin \frac{1}{2}(n \theta-t)} .
\end{aligned}
$$

The case $W_{1}=W_{+}$was considered in Example 4.1. For $n \geq 2, W_{n}$ is holomorphic on $\hat{\mathbb{C}} \backslash\{0, \infty\}$ and $\Gamma_{t}$ is defined for $-\frac{\pi}{2}<t<\frac{\pi}{2}$. For such $t, \zeta_{n}$ is computed by taking the $n$th root of the above expression for $\zeta_{n}^{n}$ in such a way that $\theta \mapsto \zeta_{n}\left(t, e^{i \theta}\right)$ is continuous; the result is a "sinusoidal" closed curve with a dihedral symmetry group of order $2 n$ generated by $e^{i \pi / n} \beta(z), \beta(z)=1 / \bar{z}$. In the context of the preceding remarks, the interpretation $W_{n}=\lambda_{\varphi *} W_{1}$ is the infinitesimal version of the idea that conjugation of $\zeta_{1}$ by $\varphi(z)=z^{1 / n}$ yields $\lambda_{\varphi} \zeta_{1}=\zeta_{n}$.

In the same vein, note that $\omega_{n}$ has simple poles at the $2 n$th roots of unity $p_{k}=e^{i k \pi / n}$, with residue $\operatorname{res}_{p_{k}} \omega_{n}=$ $(-1)^{k} i$; by the remark in Example 4.1, the tangent line to $\Gamma_{t}$ at the pivot point $p_{k}$ therefore has constant angular rate of rotation $\frac{d \alpha}{d t}=-i / \operatorname{res}_{p_{k}} \omega_{n}=(-1)^{k+1}$. Using $\zeta_{n}=\lambda_{\varphi} \zeta_{1}$, the fact that the rotation rates are independent of $n$ may be regarded as an obvious consequence of conformal invariance.

Figure 6 shows $\Gamma_{t}$ in the case $n=3$, for positive times only. For $-\pi / 2<t<\pi / 2$, each arch of $\Gamma_{t}$ sweeps out a sector of $\hat{\mathbb{C}} \backslash\{0, \infty\}$, approaching perpendicularity to $S^{1}$ at its endpoints as $t \rightarrow \pm \frac{\pi}{2}$. (Were we to abandon regularity, $\Gamma_{t}$ could be regarded as a $\pi$-period geodesic, passing repeatedly through the singular curve $\Gamma_{\pi / 2}$ consisting of $n$ straight lines intersecting at $0, \infty$.)

\section{GEODESICS GENERATED BY RATIONAL VECTOR FIELDS}

In this section we show how to represent all possible short-time behavior of geodesics - up to conformal equivalence near $S^{1}$-in the form $\Gamma_{t}=\exp (t W)$, where $W$ is a rational vector field. Before presenting a comprehensive approach to this problem, we discuss some special constructions that are useful for generating further concrete examples. First we observe that the highly symmetrical geodesics $\exp \left(t W_{n}\right)$ in Example 6.2 may be modulated to give arbitrary rotation rates $d \alpha_{k} / d t=-i / \operatorname{res}_{p_{k}} \omega$ (of alternating sign) at the pivot points $p_{k} \in S^{1}$. Since $\omega_{n}=i n d \theta / \sin n \theta$ has simple poles at the $2 n$th roots of unity $p_{k}=e^{i k \pi / n}$, it suffices to multiply $\omega_{n}$ by a function $J(z)$ that is positive on $S^{1}$ with prescribed values $J\left(p_{k}\right)>0$. In fact, suitable products $\tilde{\omega}_{n}=J \omega_{n}$ may be obtained within the class of rational differentials using methods of trigonometric interpolation. Namely, consider the Fejér kernel with index $\mu=2 n-1$ :

$$
\begin{aligned}
\mathcal{K}(\theta) & =\frac{2}{\mu+1}\left\{\frac{\sin \frac{1}{2}(\mu+1) \theta}{2 \sin \frac{1}{2} \theta}\right\}^{2}=\frac{1}{4 n} \frac{1-\cos 2 n \theta}{1-\cos \theta} \\
& =K\left(e^{i \theta}\right), \\
K(z) & =\frac{1}{4 n} \frac{\left(z^{2 n}-1\right)^{2}}{z^{2 n-1}(z-1)^{2}}=\frac{1}{4 n} z^{1-2 n}\left\{\sum_{j=0}^{2 n-1} z^{j}\right\}^{2} .
\end{aligned}
$$

Note that $K(z)$ is analytic and real on $S^{1}$, with special values $K(1)=n$ and $K\left(p_{k}\right)=0, k \neq 0$. Thus, a linear combination

$$
\mathcal{J}(\theta)=\frac{1}{n} \sum_{j=0}^{2 n-1} \lambda_{j} \mathcal{K}\left(\theta-\frac{j \pi}{n}\right)
$$

satisfies $\mathcal{J}(k \pi / n)=\lambda_{k}$, and may be conveniently used to interpolate real periodic functions $f(\theta)$ at equally spaced points; using $\lambda_{j}=f(j \pi / n)$, the interpolating function $\mathcal{J}(\theta)$ is known as the Jackson polynomial of $f(\theta)$ (of in$\operatorname{dex} \mu=2 n-1)$.

For present purposes, the key advantage of Jackson interpolation (over, say, Fourier-Lagrange interpolation) is that $\mathcal{J}$ is uniformly bounded by the extreme interpolation values $\min \left\{\lambda_{j}\right\} \leq \mathcal{J}(\theta) \leq \max \left\{\lambda_{j}\right\}$. (For a proof of this and for other relevant background, we refer the reader to [Zygmund 59], especially pp. 21-22.) In particular, if all interpolation values are positive, so is $\mathcal{J}(\theta)$. 
The rational vector field dual to $\tilde{\omega}_{n}=J \omega_{n}$ is thus analytic along $S^{1}$ and defines an element $\tilde{W}_{n}=W_{n} / J \in$ $T_{\circ} \Lambda$; it has precisely the same zeros $p_{k}$ along $S^{1}$ as $W_{n}$, but with magnitudes of the $2 n$ rotation rates now fully controlled by the coefficients $\lambda_{j}>0$.

Example 7.1. (Modulated sinusoidal variation.) We have already used the Jackson polynomial of index $\mu=1$ (implicitly in Example 5.4),

$$
\begin{aligned}
& \mathcal{J}(\theta)=1+\frac{2 r}{\left(r^{2}+1\right)} \cos \theta=J\left(e^{i \theta}\right), \\
& J(z)=1+\frac{r\left(z^{2}+1\right)}{z\left(r^{2}+1\right)}, \quad r>1 .
\end{aligned}
$$

This time we consider the parameter range $-1<r<1$, for which the ratio

$$
\frac{J(1)}{J(-1)}=\left(\frac{r+1}{r-1}\right)^{2}
$$

achieves all positive values. The product

$$
\begin{aligned}
\tilde{\omega}_{1} & =J \omega_{1}=\omega_{1}+\frac{2 i r\left(z^{2}+1\right) d z}{\left(r^{2}+1\right) z\left(z^{2}-1\right)} \\
& =\frac{i d \theta}{\sin \theta}+\frac{2 i r}{\left(r^{2}+1\right)} \cot \theta d \theta
\end{aligned}
$$

may therefore be scaled by $1 / c>0$ to give any desired negative and positive rotation rates $\frac{d \alpha}{d t}=\mp c / J( \pm 1)$ at the simple poles $z= \pm 1$, respectively. Also, $\tilde{\omega}_{1}$ has simple zeros at $-r,-1 / r$, which eventually disrupt the smooth evolution of $\Gamma_{t}$. Setting $c=1$ for convenience, the time it takes to reach $-1 / r$ may be computed as the potential difference

$$
\Delta U=U\left(-\frac{1}{r}\right)-U(i)=\frac{\pi}{2} \frac{(1-r)^{2}}{1+r^{2}},
$$

where $U$ is the real part of the complex potential

$$
\phi=i \log \frac{z-1}{z+1}+\frac{2 i r}{r^{2}+1} \log \frac{z^{2}-1}{z} .
$$

In the same time interval, the net rotation of $\Gamma_{t}$ at $z=-1$ is

$$
\Delta \theta=\frac{\Delta U}{J(-1)}=\frac{\pi}{2}
$$

This result is evident in Figure 7, which shows $\Gamma_{t}$ for $r=\frac{1}{2}$ on the positive time interval $0 \leq t<\Delta U=\pi / 10$.

Remark 7.2. The length $\mathcal{L}=\int_{S^{1}}|\omega|$ for such a meromorphic differential $\tilde{\omega}_{n}=J \omega_{n}$ is infinite. On the other

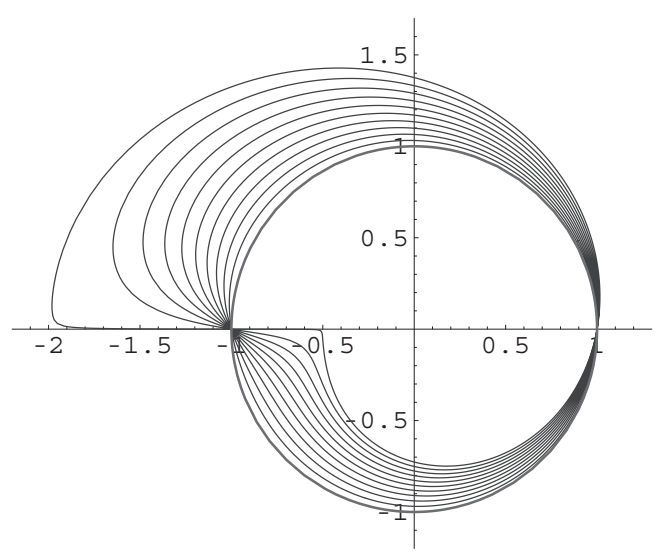

FIGURE 7. Geodesic with unequal pivot rates at $z= \pm 1$.

hand, the alternative expression $\mathcal{L}=\int_{S^{1}}-i \omega$ may still be assigned a finite value as a Cauchy principal value integral $\mathcal{L}=\operatorname{PV}\left\{\int_{S^{1}}-i \omega\right\}$, obtained by passing to the limit $\epsilon \rightarrow 0$ in the integral of $\omega$ along $S^{1}$, omitting arcs $\theta_{j}-\epsilon<\theta<\theta_{j}+\epsilon$ about each pole $p_{j}=e^{i \theta_{j}}$. By this definition, $\mathcal{L}\left(\tilde{\omega}_{n}\right)$ is easily seen to vanish, by evenness of $\mathcal{K}(\theta)$ and oddness of $\omega_{n}$ in the variables $\theta_{k}=(\theta-k \pi / n)$; one could say that the examples constructed thus far are balanced with respect to inward/outward variation from $S^{1}$.

This nongenericity may be addressed as well using trigonometric interpolation. Specifically, one may multiply $\omega_{n}$ instead by an appropriate Jackson polynomial of index $\mu=4 n-1$, choosing the "new" coefficients so that all the negative extrema of $\frac{1}{\sin n \theta}$ get multiplied by $\lambda$, say, and the positive extrema by $2 \lambda$. We omit details of the computation of $\mathcal{L}$ in terms of $\lambda$, showing that any $\mathcal{L} \in \mathbb{R}$ may be achieved by this method. The main point is that $\mathcal{L}=\operatorname{PV}\left\{\int_{S^{1}}-i \omega\right\}$ equals the real part of the integral over a slightly smaller circle,

$$
\mathcal{L}=\Re\left(\int_{|z|=1-\epsilon}-i \omega\right)
$$

(as explained in Section 9), and may therefore be computed by residue calculus. Actually, the most important feature of the latter integral is that it is meaningful even when $\omega$ has higher-order poles, as required below.

We turn now to the general problem of rational representation of analytic vector fields $W \in T_{0} \Lambda$ up to conformal equivalence near $S^{1}$. While the latter are normal along $S^{1}$, it suffices (by $W \leftrightarrow i W$ ) to represent equivalence classes of tangent fields, i.e., orbits $\operatorname{Ad}_{H} W$. Further, duality $\left(W=w(\theta) \partial_{\theta} \leftrightarrow \omega=d \theta / w(\theta)\right)$ reduces the problem to achieving all possible meromorphic data along $S^{1}$ for nonvanishing, real meromorphic differentials 
on $S^{1}$. Namely, using rational differentials of this kind, we need to realize prescribed pole orders $\mu_{j} \in \mathbb{Z}^{-}$and residues $\nu_{j} \in \mathbb{R}$, along with given $S^{1}$-period $\mathcal{I}=\Re\left(\int_{\gamma_{\epsilon}} \omega\right)$ (corresponding to the integral $\mathcal{L}$ of the above remark). Finally, we need to match specified polarity for the $j$ th pole,

$$
\sigma_{j}= \pm 1=\operatorname{sgn} w(\theta)\left(\theta-\theta_{j}\right)^{\mu_{j}}, \quad \theta \text { near } \theta_{j}
$$

This "sign" invariant enables us to conveniently express the polarity constraints (9-2) restricting the above set of data. Note that residues of consecutive simple poles must have opposite sign. For reading the following proof of Theorem 7.3, one may refer as needed to Sections 9 and 10 for fuller discussion of above data and the relevant classification result, Theorem 10.2.

Theorem 7.3. For $W=w(z) \frac{\partial}{\partial z} \in T_{\Gamma_{\circ}} \Lambda$, the geodesic departing from $\Gamma_{\circ}$ with velocity $W$ is representable in the form $\exp (t W)=h(\exp (t V))$, for small $|t|$, with $h \in H$ and rational vector field

$$
V=\frac{a_{m} z^{m}+\cdots+a_{0}}{b_{n} z^{n}+\cdots+b_{0}} \frac{\partial}{\partial z},
$$

which can be constructed explicitly using meromorphic data of

$$
\omega=\frac{d z}{w(z)}
$$

the dual differential to $W$.

Proof: To construct the required rational differential

$$
\omega=w(\theta) d \theta=\frac{\mathbf{w}(z) d z}{i z},
$$

we begin by assigning pole positions $p_{1}=e^{i \theta_{1}}, \ldots, p_{m}=$ $e^{i \theta_{m}}$ in counterclockwise order around $S^{1}$, as follows. Note that there is an even number, $2 N$, of prescribed odd-order poles, by (9-2). Assuming $N>0$, we space these poles evenly around the circle at angles $\theta_{j_{1}}=$ $\pi / N, \ldots, \theta_{j_{2 N}}=2 \pi$. The exact locations of even-order poles will play no role; only the counterclockwise order must be respected.

We construct $\omega$ as a sum $\omega=\omega_{s}+\omega_{r}$ of singular and regular parts. Then $\omega_{s}=w_{s}(\theta) d \theta$ will be a linear combination of the following basic differentials (and " $\theta$ - translates" of these):

$$
\begin{aligned}
\alpha & =\frac{1}{2} \cot \frac{\theta}{2} d \theta=\frac{(z+1) d z}{2(z-1) z}, \\
\beta^{-(2 k+1)} & =\csc \theta\left(\cot \frac{\theta}{2}\right)^{2 k} d \theta \\
& =2(-1)^{k} \frac{(z+1)^{2 k-1} d z}{(z-1)^{2 k+1}}, k=1,2, \ldots, \\
\beta^{-2 k} & =\frac{d \theta}{(1-\cos \theta)^{k}}=-i(-2)^{k} \frac{z^{k-1} d z}{(z-1)^{2 k}}, \\
k & =1,2, \ldots
\end{aligned}
$$

Note that $\alpha$ has a simple pole at $z=1$ with residue $\operatorname{res}[\alpha, 1]=1$; the other finite pole $z=0$ has residue $\operatorname{res}[\alpha, 0]=-\frac{1}{2}$ and consequently $\Re\left[\int_{\gamma_{\epsilon}} \alpha\right]=0$. For each integer $\mu<-1, \beta^{\mu}$ has a pole at $z=1$ of order $\operatorname{ord}_{1} \beta^{\mu}=\mu$ and zero residue; since $\beta^{\mu}$ has no other poles, $\Re\left[\int_{\gamma_{\epsilon}} \beta^{\mu}\right]=0$. The corresponding differentials with pole $p=e^{i \theta_{j}}$ are the pullbacks, $\alpha_{j}=\rho_{j}^{*} \alpha, \beta_{j}^{\mu}=\rho_{j}^{*} \beta^{\mu}$, by the rotation $\rho_{j}(z)=e^{-i \theta_{j}} z$.

Now consider the following sum:

$$
\omega_{s}=\sum_{j=1}^{m} \sigma_{j} \beta_{j}^{\mu_{j}}+\nu_{j} \alpha_{j} .
$$

Observe that $\omega_{s}$ satisfies the data $\left\{\mu_{j}, \nu_{j}, \sigma_{j}\right\}$, but gives $\mathcal{I}=0$. To achieve nonzero $\mathcal{I}$, we may add the regular term

$$
\frac{\mathcal{I} d \theta}{2 \pi}=\frac{\mathcal{I} d z}{2 \pi i z}
$$

(In the regular case $\omega_{s}=0$, we have simply $\omega=\mathcal{I} d \theta / 2 \pi$, $\mathcal{I} \neq 0$.) The sum $\omega_{s}+\mathcal{I} d z / 2 \pi i z$ has just one defect: it may vanish at points on $S^{1}$. To correct this, assume first that the prescribed orders $\mu_{j}$ are not all even, so $N \geq 1$. Then either the regular differential $\sin N \theta d \theta$ or its opposite agrees in sign with $\omega_{s}$ in the vicinity of every pole. It follows that for $\lambda \in \mathbb{R}$ of correct sign and sufficiently large magnitude, the sum

$$
\omega_{s}+\frac{\mathcal{I} d z}{2 \pi i z}+\lambda\left(z^{N-1}-z^{-N-1}\right) d z
$$

is nonvanishing on $S^{1}$. Since $\sin N \theta d \theta$ contributes nothing to $\mathcal{I}$, we have thus satisfied all requirements. On the other hand, if $N=0$, all signs $\sigma_{j}$ agree, and the sum

$$
\sum_{j=1}^{m} \sigma_{j} \beta_{j}^{\mu_{j}}= \pm \sum_{j=1}^{m} \beta_{j}^{\mu_{j}}
$$

is seen to be nonvanishing on $S^{1}$. In this case,

$$
\omega=\frac{\mathcal{I} d z}{2 \pi i z}+\sum_{j=1}^{m} \lambda \beta_{j}^{\mu_{j}}+\nu_{j} \alpha_{j}
$$



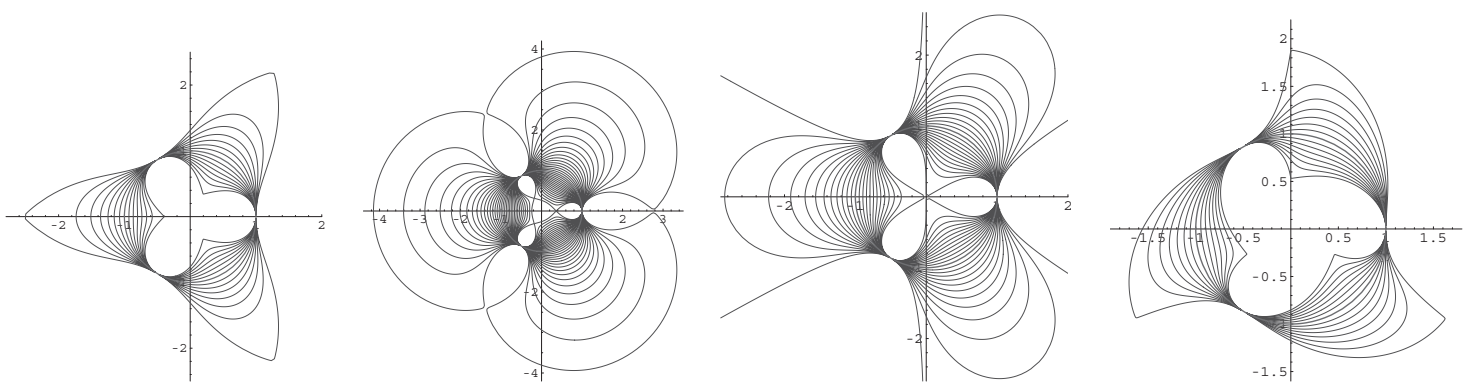

FIGURE 8. Influence of the $S^{1}$ period and residues.

does the job, with $\operatorname{sgn}(\lambda)=\operatorname{sgn}\left(\sigma_{j}\right)$, and $|\lambda|$ large enough so that the middle term dominates the other terms along $S^{1}$ and prevents unwanted zeros.

Example 7.4. (Double poles.) We consider geodesics determined by differentials $\omega$ with three second-order poles on the unit circle. To highlight the influence of residues and the $S^{1}$ period, the above differentials will be combined in a simple manner respecting threefold rotational symmetry. Namely, for $j=0,1,2$, let $\beta_{j}^{-2}=\rho_{j}^{*} \beta^{-2}$, $\alpha_{j}=\rho_{j}^{*} \alpha$, where $\rho_{j}(z)=\rho^{j} z, \rho=e^{2 \pi i / 3}$, and define

$$
\begin{aligned}
\omega & =c i d \theta+\sum_{j=0}^{2}\left(\nu i \alpha_{j}+\lambda i \beta_{j}^{-2}\right) \\
& =c \frac{d z}{z}+\nu \frac{3 i\left(z^{3}+1\right) d z}{2 z\left(z^{3}-1\right)}-\lambda \frac{18 z^{2} d z}{\left(z^{3}-1\right)^{2}} .
\end{aligned}
$$

Here, $c=\mathcal{I} / 2 \pi, \nu \in \mathbb{R}$ may be specified arbitrarily, and $\lambda>0$ is subsequently chosen large enough so that $\omega$ does not vanish on $S^{1}$ (no multiple of $\sin N \theta d \theta$ needs to be added on in this case, since $\omega$ has no odd-order poles on $S^{1}$ ). The resulting geodesic moves outward from $S^{1}$ in positive time and inward in negative time, while maintaining tangency to $S^{1}$ at the three cube roots of unity.

Four cases of this construction are shown in Figure 8, each with $\lambda=1$, the first three with $\nu=0$. In the upper left, we have also set $c=0$, while the upper right and lower left show, respectively, $c>0(c \approx 0.93)$ and $c<0$ $(c \approx-0.97)$. Finally, dihedral symmetry is broken in the lower right with nonzero residues $\nu i(\nu \approx 1.75)$ and $c=0$. (We recall that $\nu$ controls the rate of change of the Kasner invariant associated with the horn angle formed by $\Gamma_{t}$ and $\Gamma_{0}=S^{1}$ at the points of tangency $\rho^{j}$ [Calini and Langer 03], but this influence is visually subtle.) The same time step has been used in each case, but the total time it takes to run into singularities increases with $c$ and $|\nu|$. This accounts for the wide variation in number of curves displayed (and scales used).

\section{ANALYTIC DIFFEOMORPHISMS OF $S^{\mathbf{1}}$}

Reflection of the extended complex plane $\hat{\mathbb{C}}$ in $S^{1}, z \mapsto$ $\breve{z}=1 / \bar{z}=R z$, restricts to an involution on the group $\mathbb{C}^{\times}=\mathbb{C} \backslash\{0\}$ with fixed-point set $S^{1}=\left\{z \in \mathbb{C}^{\times}: \breve{z}=z\right\}$. We consider a symmetric connected domain $U=\breve{U} \subset \hat{\mathbb{C}}$, and the group $\mathcal{M}(U)$ of meromorphic functions $g: U \rightarrow$ $\hat{\mathbb{C}}, g$ not identically zero. Then $R$ induces reflection of functions in $S^{1}$ by $g \mapsto \breve{g}=\mathcal{R}(g)=R \circ g \circ R$, and $\mathcal{R}: \mathcal{M}(U) \rightarrow \mathcal{M}(U)$ is an involutive automorphism with respect to the group operation of pointwise multiplication. In this sense, $\mathcal{R}$ behaves like reflection of functions in $\hat{\mathbb{R}}, g(z) \mapsto \bar{g}(z)=\overline{g(\bar{z})}$. (The latter is computationally simpler in that it also satisfies $\overline{g+h}=\bar{g}+\bar{h}$ and consequently is given by conjugation of coefficients: $\sum a_{n}\left(z-z_{0}\right)^{n} \mapsto \sum \bar{a}_{n}\left(z-\bar{z}_{0}\right)^{n}$.)

Moreover, $\mathcal{R}$ fixes $g \in \mathcal{M}(U)$ if and only if $g$ belongs to the subgroup of circle-preserving functions

$$
\begin{aligned}
\mathcal{M}_{\mathcal{R}}(U) & =\{g \in \mathcal{M}(U): \breve{g}=g\} \\
& =\left\{g \in \mathcal{M}(U): g\left(S^{1}\right) \subset S^{1}\right\}
\end{aligned}
$$

Any $g \in \mathcal{M}(U)$ yields an element $\varphi \in \mathcal{M}_{\mathcal{R}}(U)$ by the formula

$$
\varphi(z)=z g(z) \breve{g}(z)=z \frac{g(z)}{\overline{g(1 / \bar{z})}},
$$

and $g \mapsto \varphi=\Phi(g)$ defines a mapping of $\mathcal{M}(U)$ into (onto, as it turns out) the odd-degree part of $\mathcal{M}_{\mathcal{R}}(U)$; here, degree is defined by regarding $\varphi$ as a self-map of the circle. Since we are presently interested in diffeomorphisms $\varphi \in H$, it will suffice to generate maps of degree one by (8-1) using $g \in \mathcal{M}(U)$ with zero winding number.

Formula (8-1) provides a convenient way to discuss the interpolation problem for $H$, as we now demonstrate. An $N$-point configuration will refer to an $N$-tuple $\sigma=\left(\sigma_{1}, \ldots, \sigma_{N}\right)$ of $N$ distinct points in $S^{1} \subset \mathbb{C}$ in counterclockwise order on $S^{1}$. The space $\Sigma_{N}=\{\sigma\} \subset T^{N}$ of such configurations will be topologized as a subset of the $N$-torus. Let $\operatorname{Diff}_{+}^{\omega}\left(S^{1}\right)$ act componentwise on $\Sigma_{N}$, i.e., $\varphi \cdot \sigma=\left(\varphi\left(\sigma_{1}\right), \ldots, \varphi\left(\sigma_{N}\right)\right)$. 
Given two such configurations $\sigma=\left(\sigma_{1}, \ldots, \sigma_{N}\right), \tilde{\sigma}=$ $\left(\tilde{\sigma}_{1}, \ldots, \tilde{\sigma}_{N}\right)$, we first show how to construct a rational function $\varphi \in \mathcal{M}_{\mathcal{R}}(\hat{\mathbb{C}})$ satisfying $\varphi \cdot \sigma=\tilde{\sigma}$. For $1 \leq j \leq N$, let $P_{j}(z)$ be the unique polynomial of degree $(N-1)$ satisfying $P_{j}\left(\sigma_{k}\right)=\delta_{j k}$ for $1 \leq k \leq N$. Define $p \in \mathcal{M}(\hat{\mathbb{C}})$ by

$$
p(z)=\sum_{j=1}^{N} \lambda_{j} P_{j}(z), \quad \lambda_{j}= \pm \sqrt{\tilde{\sigma}_{j} \sigma_{j}^{-1}}
$$

(for the moment, either sign for $\lambda_{j}$ will do). Now consider $\varphi=\Phi(p) \in \mathcal{M}_{\mathcal{R}}(\hat{\mathbb{C}})$. Since $p\left(\sigma_{k}\right)=\lambda_{k}$ and $\breve{p}\left(\sigma_{k}\right)=$ $\breve{p}\left(\breve{\sigma}_{k}\right)=\breve{\lambda}_{k}=\lambda_{k}$, we have $\varphi\left(\sigma_{k}\right)=\sigma_{k} p\left(\sigma_{k}\right) \breve{p}\left(\sigma_{k}\right)=$ $\sigma_{k} \lambda_{k}^{2}=\tilde{\sigma}_{k}$, as required.

Proposition 8.1. Let $\operatorname{Diff}_{+}^{\omega}\left(S^{1}\right)$ be the group of analytic diffeomorphisms $\varphi(z)$ of $S^{1}$. For each $N$, Diff ${ }_{+}^{\omega}\left(S^{1}\right)$ acts transitively on the space of $N$-point configurations $\Sigma_{N}$.

Proof: By a standard argument, it suffices to show that Diff ${ }_{+}^{\omega}\left(S^{1}\right)$-orbits are open in $\Sigma_{N}$, for the connected space $\Sigma_{N}$ is a disjoint union of its Diff ${ }_{+}^{\omega}\left(S^{1}\right)$-orbits, of which there can therefore be only one.

Thus, we fix a configuration $\sigma \in \Sigma_{N}$, and consider nearby $\tilde{\sigma} \in V \subset \Sigma_{N}$, where the small neighborhood $V$ of $\sigma$ will be chosen according to the following considerations. As long as $\tilde{\sigma}_{k}$ never equals $-\sigma_{k}$, for any $k$, all signs in (8-2) may be defined using the principal branch of the square root $\lambda_{k}=\sqrt{\tilde{\sigma}_{k} / \sigma_{k}}$, an element of the right half-plane, so as to vary continuously with $\tilde{\sigma} \in V$. By this choice, the special case $\sigma=\tilde{\sigma}$ yields $\lambda_{k}=1$, for all $k$; hence $p(z)=\sum_{j=1}^{N} P_{j}(z) \equiv 1$ and $\Phi(p)=$ Id. Further, as $\tilde{\sigma} \in V$ varies, the resulting function $p(z)=\sum_{j=1}^{N} \lambda_{j} P_{j}(z)$ varies continuously in the $C^{1}\left(S^{1}\right)$-topology. In fact, the assignment $\tilde{\sigma} \mapsto \mu(\tilde{\sigma})=\varphi=\Phi(p) \in C^{1}\left(S^{1}\right)$ is continuous, so $\mu(V)$ may be assumed (by taking $V$ small enough) to lie in $\operatorname{Diff}_{+}^{\omega}\left(S^{1}\right)$, indeed, within any given $C^{1}\left(S^{1}\right)$ neighborhood of the identity $\mu(\sigma)=\operatorname{Id} \in \operatorname{Diff}_{+}^{\omega}\left(S^{1}\right)$. Since $\tilde{\sigma}=\mu(\tilde{\sigma}) \cdot \sigma$, it follows that the orbit $\operatorname{Diff}_{+}^{\omega}\left(S^{1}\right) \cdot \sigma$ contains the neighborhood $V=\mu(V) \cdot \sigma$ of $\sigma$. Since $\sigma \in \Sigma_{N}$ was arbitrary, we conclude that $\operatorname{Diff}_{+}^{\omega}\left(S^{1}\right)$-orbits are open.

\section{REAL MEROMORPHIC DIFFERENTIALS ON $S^{1}$}

A meromorphic differential on $S^{1}$ will refer either to a meromorphic differential $\omega=f(z) d z$ defined on

$$
A_{\epsilon}=\{z \in \mathbb{C}: 1-\epsilon<|z|<1+\epsilon\}
$$

for some $\epsilon>0$, or to the restriction of such an $\omega$ to $S^{1}$. It will be convenient to confuse these two slightly different objects, taking full advantage of the fact that each determines the other. Indeed, we will abuse notation by writing

$$
\omega=\mathbf{w}(z) \frac{d z}{i z}=w(\theta) d \theta
$$

The two expressions for $\omega$ are related by a formal change of variables $z=e^{i \theta}, d z=i e^{i \theta} d \theta, w(\theta)=\mathbf{w}\left(e^{i \theta}\right)$, with $\mathbf{w}(z)$ the analytic continuation of $\mathbf{w}\left(e^{i \theta}\right)$ to $A_{\epsilon}$. One may prefer to write

$$
z=r e^{i \theta}, \quad d z=i r e^{i \theta} d \theta+e^{i \theta} d r
$$

and subsequently set $r=1$ and $d r=0$ when restricting to tangent vectors $v \partial_{\theta}$ along $S^{1}$. In rectangular coordinates, one drops the imaginary part of

$$
\frac{d z}{i z}=\frac{d x+i d y}{i(x+i y)}=\frac{x d y-y d x}{x^{2}+y^{2}}+i \frac{x d x+y d x}{x^{2}+y^{2}}
$$

when restricting to tangent vectors $v\left(x \partial_{y}-y \partial_{x}\right)$. The original analytic differential $\mathbf{w} \frac{d z}{i z}=\alpha+i \star \alpha$ may be recovered from the harmonic differential

$$
\alpha=\mathbf{w} \frac{x d y-y d x}{x^{2}+y^{2}}
$$

via the operation of star conjugation [Springer 57],

$$
\star(a d x+b d y)=-b d x+a d y .
$$

The singularities of $\omega$ are the zeros and poles of $\omega=\mathbf{w} d z / i z$ on $S^{1}$. It will be assumed henceforth that $\omega$ is not identically zero, so singularities are isolated. The order of $\omega$ at a point $p=e^{i \theta}$ is the integer $\mu=\operatorname{ord}_{p} \omega$ in the local representation $\omega=(z-p)^{\mu} g(z) d z$, with $g(z)$ analytic and nonvanishing at $p$. For fixed $\omega$, the value $\operatorname{ord}_{p} \omega$ of the function ord $\omega: S^{1} \rightarrow \mathbb{Z}$ is positive, negative, or zero, depending on whether $p$ is respectively a zero, pole, or regular point of $\omega$. The residue $\nu \in \mathbb{C}$ of $\omega$ at $p=e^{i \theta}$ is well defined by

$$
\nu=\operatorname{res}_{p} \omega=\frac{1}{2 \pi i} \int_{c_{\epsilon}(p)} \omega,
$$

where $c$ is a positively oriented circle of sufficiently small radius $\epsilon$ centered at $p ; \nu=a_{-1}$ in the local Laurent series representation $\omega=\sum_{n=N}^{\infty} a_{n}(z-p)^{n} d z$. In particular, $\nu=\operatorname{res}_{p} \omega$ vanishes except at the finitely many poles $p_{j_{1}}, \ldots, p_{j_{n}} \in S^{1}$.

We also associate to such $\omega$ the $S^{1}$-period $\int_{\gamma_{\epsilon}} \omega$ obtained by integrating around a slightly smaller circle, $\gamma_{\epsilon}=\left(1-\frac{\epsilon}{2}\right) e^{i \theta}$. Finally, if $\omega$ has zeros $p_{k_{1}}, \ldots, p_{k_{m}} \in S^{1}$, 
assumed to be indexed in counterclockwise cyclic order, we define subperiods $\int_{\gamma_{s}} \omega$ by integrating along a suitable path $\gamma_{s}$ joining $p_{k_{s}}$ to $p_{k_{s+1}} ; \gamma_{k}$ starts at $p_{k_{s}}$, goes radially inward a distance $\epsilon / 2$, then counterclockwise along a portion of $\gamma_{\epsilon}$, then radially out to $p_{k_{s+1}}$. When $m>0$, the sum of subperiods equals the $S^{1}$-period.

Of particular interest will be the real meromorphic differentials $\omega=w(\theta) d \theta$ on $S^{1}$; here $w(\theta) \in \hat{\mathbb{R}}=\mathbb{R} \cup\{\infty\}$ for all $\theta$, and $w(\theta)$ may be regarded as a real analytic function on $S^{1}$ (helping to motivate the above notation). In this case, the residues $\nu_{1}, \ldots, \nu_{n}$ are automatically real; further, these values determine the imaginary parts $\Im\left[\int_{\gamma_{\epsilon}} \omega\right], \Im\left[\int_{\gamma_{s}} \omega\right]$ of the above integrals. These claims follow, via Cayley transformation

$$
w=\frac{z-i}{z+i}, \quad z=i \frac{w+1}{1-w}
$$

from corresponding facts for real meromorphic differentials on the extended real line $\hat{\mathbb{R}}$.

Alternatively, the antiholomorphic involution in the Riemann sphere $\hat{\mathbb{C}}$ given by reflection in the unit circle $z \mapsto \breve{z}=1 / \bar{z}$ may be used-just as well as complex conjugation - to define a real structure on $\hat{\mathbb{C}}$ and associated spaces. Thus, one may conjugate functions $f(z)$, and differentials $\omega=f(z) d z$ according to the formulas

$$
\begin{aligned}
f^{*}(z) & =\frac{1}{\breve{f}(z)}=\overline{f\left(\frac{1}{\bar{z}}\right)}, \\
\omega^{*} & =f^{*}(z) d z^{*}=-f^{*}(z) \frac{d z}{z^{2}} .
\end{aligned}
$$

Unlike star conjugation (which satisfies $\star \star \omega=-\omega$ ), $\omega \mapsto$ $\omega^{*}$ is an involution and behaves formally like conjugation with respect to the real line: $\bar{\omega}=\bar{f}(z) d z=\overline{f(\bar{z})} d z$. In particular, if $\gamma^{*}=\breve{\gamma}$ is the reflection of an oriented curve $\gamma$ in $S^{1}$, then one directly verifies that $\int_{\gamma^{*}} \omega^{*}=\overline{\int_{\gamma} \omega}$.

In the case of a real differential $\omega=w(\theta) d \theta=\mathbf{w}(z) \frac{d z}{i z}$, we have $\omega^{*}=\omega$. For curves satisfying $\gamma^{*}=-\gamma$, e.g., for a small circle meeting $S^{1}$ orthogonally, it follows that $\int_{\gamma} \omega=-\int_{\gamma^{*}} \omega^{*}=-\overline{\int_{\gamma} \omega}$ is imaginary; thus, any residues of $\omega$ on $S^{1}$ must be real, as claimed above. Similarly, by taking a contour $\gamma$ that mostly follows $S^{1}$ but makes small inward detours around the poles $p_{j_{r}}$, one obtains

$$
\begin{aligned}
2 i \Im\left[\int_{\gamma_{\epsilon}} \omega\right] & =2 i \Im\left[\int_{\gamma} \omega\right] \\
& =\int_{\gamma} \omega-\int_{\gamma^{*}} \omega^{*} \\
& =-2 \pi i \sum \operatorname{res}\left[\omega ; p_{j_{r}}\right] .
\end{aligned}
$$

The quantities $\Im\left[\int_{\gamma_{s}} \omega\right]$ are handled similarly. In view of these facts, the $S^{1}$-period $\mathcal{I}$ and subperiods $\mathcal{I}_{s}$ of a real meromorphic differential on $S^{1}$ will refer henceforth to the respective real parts $\mathcal{I}=\Re\left[\int_{\gamma_{\epsilon}} \omega\right]$ and $\mathcal{I}_{s}=\Re\left[\int_{\gamma_{s}} \omega\right]$.

For such $\omega$ with no poles on $S^{1}$, we have $\mathcal{I}=\int_{S^{1}} \omega$. More generally, if $\omega$ has at most simple poles on $S^{1}$, then $\mathcal{I}$ may be computed as $\mathcal{I}=\operatorname{PV}\left\{\int_{S^{1}} \omega\right\}$ - the Cauchy principal value integral defined by passing to the limit $\epsilon \rightarrow 0$ in the integral of $\omega$ along $S^{1}$, omitting arcs $\theta_{j}-\epsilon<\theta<\theta_{j}+\epsilon$ about each pole $p_{j}=e^{i \theta_{j}}$. Such integrals are discussed, for example, in [Ablowitz and Fokas 97], where one may also find the following elementary result: If $f(z) d z$ has a simple pole at $z_{0}$ and $C(\epsilon)$ denotes a circular arc centered at $z_{0}$ with angular measure $\phi$ and (variable) radius $\epsilon$, then

$$
\lim _{\epsilon \rightarrow 0} \int_{C(\epsilon)} f(z) d z=i \phi \operatorname{res}\left[f(z) d z, z_{0}\right] .
$$

This may be applied in the present context by deforming $\gamma_{\epsilon}$ to a new contour $\tilde{\gamma}_{\epsilon}$ that coincides with $S^{1}$ except for "inward" detours $C_{j}(\epsilon)$ around the poles, $C_{j}(\epsilon)$ being nearly half of a circle of radius $\epsilon$, centered at $p_{j}$. In the limit $\epsilon \rightarrow 0$, the detours contribute the imaginary part of $\int_{\tilde{\gamma}_{\epsilon}} \omega$ (the residues of $\omega$ being real), while the real part is given by the above Cauchy principal value integral over $S^{1}$. Thus, we have heuristically explained the term $S^{1}$ period (though the Cauchy principal value interpretation of $\mathcal{I}$ is no longer valid when $\omega$ has higher-order poles).

We need to consider one additional type of data for such $\omega=w(\theta) d \theta$, the polarity (or sign) of the $j$ th singularity $p_{j}=e^{i \theta_{j}}$ : for $\theta$ near $\theta_{j}$, the sign is given by $\sigma_{j}= \pm 1=\operatorname{sgn} w(\theta)\left(\theta-\theta_{j}\right)^{\mu_{j}}$. In particular, the polarity of a simple pole agrees with the sign of the residue, as one may easily verify. Further, the polarity of any singularity is invariant under orientation-preserving analytic diffeomorphisms of $S^{1}$.

Now observe that the sign of the real function $w(\theta)$ changes precisely at odd-order singularities. Consequently, the sequence $\sigma_{1}, \sigma_{2}, \ldots, \sigma_{m}$ jumps up or down precisely at odd-order singularities as all singularities are visited in counterclockwise order. If there is at least one simple pole, it follows that the sequence $\left\{\sigma_{j}\right\}$ is uniquely determined by the values $\left\{\mu_{j}\right\},\left\{\nu_{j}\right\}$; otherwise, the sequence is determined only up to an overall factor \pm 1 . The data $\left\{\mu_{j}, \nu_{j}, \sigma_{j}\right\}$ for a real meromorphic differential $\omega=w(\theta) d \theta$ on $S^{1}$ is subject to the following two polarity constraints:

$$
\sigma_{j}=(-1)^{\mu_{j}} \sigma_{j-1}, \quad \mu_{j}=-1 \Rightarrow \sigma_{j}=\operatorname{sgn} \nu_{j} .
$$


Note that (9-2) imposes constraints even on the abridged data $\left\{\mu_{j}, \nu_{j}\right\}$; for example, the sum $\sum_{j=1}^{m} \mu_{j}$ must be even, and the residues $\nu_{j}, \nu_{j+1}$ at two adjacent simple poles have opposite sign.

\section{EQUIVALENCE OF REAL MEROMORPHIC DIFFERENTIALS ON $S^{1}$}

As shown in [Moser 65], two smooth volume forms $\omega$ and $\tilde{\omega}$ on a compact, connected manifold $M$ are equivalentthey are related by pullback, $\omega=h^{*} \tilde{\omega}$, under an orientation-preserving diffeomorphism $h$ of $M$-if and only if $\omega$ and $\tilde{\omega}$ have the same volume $\int_{M} \omega=\int_{M} \tilde{\omega}$. (The necessary condition follows at once from the change of variables formula.) The second of the two main arguments used in [Moser 65] (the "Hodge-theoretic" one) may be adapted to the present context of real meromorphic differentials on $S^{1}$, as we now demonstrate.

Lemma 10.1. Let $\omega_{r}=w_{r} d \theta, r=0,1$, be two real meromorphic differentials on $S^{1}$ satisfying:

(1) The ratio $q(z)=\omega_{1} / \omega_{0}$ defines a positive analytic function on $S^{1}$; i.e., $\omega_{0}, \omega_{1}$ have the same singularities $p_{1}, \ldots, p_{m} \in S^{1}$, with equal orders $\mu_{j}$ and polarities $\sigma_{j}$.

(2) $\omega_{0}, \omega_{1}$ have equal residues $\nu_{j}, S^{1}$-periods $\mathcal{I}=$ $\Re\left[\int_{\gamma_{\epsilon}} \omega_{r}\right]$, and subperiods $\mathcal{I}_{k}=\Re\left[\int_{\gamma_{k}} \omega_{r}\right]$ (in the event there are zeros).

Then $\omega_{0}$ and $\omega_{1}$ are analytically equivalent, in fact, diffeotopic: the linear isotopy of differentials, $\omega_{t}=t \omega_{1}+$ $(1-t) \omega_{0}$, is realized by pullback, $\omega_{0}=h_{t}^{*} \omega_{t}$, via a diffeotopy $h_{t}:[0,1] \times S^{1} \rightarrow S^{1}$.

Proof: Each intermediate $\omega_{t}=w_{t} d \theta$ in the isotopy is of the same type and shares the same data as $\omega_{0}, \omega_{1}$. In particular, the quotient $q_{t}=\omega_{t} / \omega_{0}$ defines, for each fixed $t \in[0,1]$, a positive, real analytic function on $S^{1}$. By (2), the time derivative $\dot{\omega}=\frac{d}{d t} \omega_{t}=\omega_{1}-\omega_{0}$ has vanishing periods on $A_{\epsilon} \backslash P$, an annular neighborhood of $S^{1}$ with polar set of $\omega_{t}$ removed. Thus, $\dot{\omega}$ is exact and may be expressed as $\dot{\omega}=d \alpha$ for some real meromorphic function $\alpha: S^{1} \rightarrow \mathbb{R} \cup\{\infty\}$. In case there are zeros, we choose $\alpha$ to vanish at one of them; hence, by (2), it vanishes on all zeros of $\omega_{t}$.

Differentiating the desired equation $\omega_{0}=h_{t}^{*} \omega_{t}$ and suppressing the $t$ subscripts, we obtain

$0=\frac{d}{d t} h^{*} \omega=\frac{d}{d t} h^{\prime} w \circ h d \theta=\left[\dot{h}^{\prime} w \circ h+h^{\prime} \dot{w} \circ h+h^{\prime} w^{\prime} \circ h \dot{h}\right] d \theta$.
Using $[\alpha \circ h]^{\prime}=\alpha^{\prime} \circ h h^{\prime}=h^{\prime} \dot{w} \circ h$ to replace the middle term, the above equation may be expressed more compactly as $0=[\dot{h} w \circ h+\alpha \circ h]^{\prime}$. In fact, we aim to make the bracketed quantity itself vanish, that is, to solve the following ODE:

$$
\frac{d h}{d t}=-\frac{\alpha(h)}{w(h)}, \quad h_{0}=\mathrm{Id} .
$$

Assuming that (10-1) has been solved for analytic diffeomorphisms $h_{t}, 0 \leq t \leq 1$, we then have $h_{t}^{*} \omega_{t}=$ const and $\omega_{0}=h_{0}^{*} \omega_{0}=h_{1}^{*} \omega_{1}$, as required.

To show that the above ODE is suitably well behaved (and to see why all hypotheses are needed), consider the meromorphic differentials $\eta=-\omega / \alpha, 0 \leq t \leq 1$, at singular and nonsingular points of $\alpha$. If $z \in S^{1}$ is not a zero or pole of $\alpha$, then the above assumptions imply that $\eta \neq 0$ at $z$. On the other hand, if $z \in S^{1}$ is a zero or pole of $\alpha$, then $d \alpha / \alpha$ has a (simple) pole at $z$. Since the quotient

$$
\frac{\omega}{d \alpha}=\frac{\omega}{\omega_{1}-\omega_{0}}=\frac{q_{t}}{q_{1}-1}
$$

is nonvanishing on $S^{1}$ for $0 \leq t \leq 1$, it follows that $\eta=-\frac{\omega}{d \alpha} \frac{d \alpha}{\alpha}$ has a pole at $z$. We conclude that $\eta$ is nonvanishing on $S^{1}$ for $0 \leq t \leq 1$ (and one can show that $\eta$ has poles at all singularities of $\left.\omega_{t}\right)$.

Dualizing $\eta=-w d \theta / \alpha$, we thus obtain the (timedependent) real analytic vector field on $S^{1}, X=-\frac{\alpha}{w} \partial_{\theta}$, defining (10-1). By the theory of ODEs, $X$ generates an analytic diffeotopy $h$ on the compact manifold $S^{1}$ (see, for instance, [Hirsch 76] for the smooth theory, and [Cartan 63, Dieudonné 69] for analytic dependence on initial conditions).

\section{Theorem 10.2.}

(i) Two real meromorphic differentials on $S^{1}$ are equivalent if and only if they have identical orderresidue-polarity-period data, $\mu_{j}, \nu_{j}, \sigma_{j}, \mathcal{I}_{k}, \mathcal{I}$, for suitable counterclockwise orderings of singularities.

(ii) For such differentials with no zeros on $S^{1}$, all equivalence classes are represented by restriction to $S^{1}$ of rational differentials on $\hat{\mathbb{C}}$, since the latter achieve arbitrary data $\mu_{j}, \nu_{j}, \sigma_{j}, \mathcal{I}$, subject only to the polarity constraints (9-2) and, when there are no poles, $\mathcal{I} \neq 0$.

(iii) The adjoint orbits $\operatorname{Ad}_{H} A$ are characterized by data of the dual meromorphic differentials as in (ii); in particular, each is represented by restriction to $S^{1}$ of a rational vector field on $\hat{\mathbb{C}}$. 
Proof: We combine results discussed above. For the nontrivial direction of (i), suppose two differentials share data $\mu_{j}, \nu_{j}, \sigma_{j}, \mathcal{I}_{k}, \mathcal{I}$, for some counterclockwise orderings of singularities. Then by Proposition 8.1 we can pull back one of the differentials by some $h \in H$ to bring locations of corresponding singularities into agreement. Then Lemma 10.1 implies that the two differentials are equivalent (in fact they are diffeotopic).

Subsequently, (ii) follows the constructions of Section 7 , which show how all data $\mu_{j}, \nu_{j}, \sigma_{j}, \mathcal{I}$ consistent with the polarity constraints may be achieved by rational differentials that are real and nonvanishing along $S^{1}$. More-complicated constructions appear to be needed for explicit representation of differentials in the general case (which we do not need).

Finally, (ii) yields (iii) via duality

$$
A=a(\theta) \partial_{\theta} \longleftrightarrow \omega=\frac{d \theta}{a}
$$

this bijection between $\mathfrak{h}$ and nonvanishing real meromorphic differentials along $S^{1}$ intertwines $\lambda_{h *}$ and pullback by $h^{-1}$. Explicitly, if $A=a(\theta) \partial_{\theta}$ and $\tilde{A}=\operatorname{Ad}_{h} A=$ $\tilde{a}(\theta) \partial_{\theta}$, then (6-1) gives $\tilde{a}(h(\theta))=h^{\prime}(\theta) a(\theta)$; hence

$$
\omega=\frac{d \theta}{a(\theta)}=h^{\prime}(\theta) \frac{d \theta}{\tilde{a}(h(\theta))}=h^{*} \frac{d \theta}{\tilde{a}(\theta)}=h^{*} \tilde{\omega},
$$

which completes the proof.

Remark 10.3. Simple heuristics further illuminate the nature of adjoint orbits of $H$. By $(6-3), 0 \neq A \in \mathfrak{h}$ spans the kernel of $B \mapsto \operatorname{ad}_{B} A$. The one-parameter group $\left\{e^{t A}\right\}$ is the continuous part of the isotropy subgroup $H_{A}=\left\{B \in H: \operatorname{Ad}_{B} A=A\right\}$. For $A$ nonvanishing on $S^{1}$, we have $H_{A} \simeq S^{1}$; the corresponding orbit $\operatorname{Ad}_{H} A$ may be identified with the homogeneous space $H / S^{1}$, already mentioned in Section 5. On the other hand, if $A$ vanishes on $S^{1}$, its orbit looks like $H / \mathbb{R}$ or, in exceptional cases, $H /\left(\mathbb{R} \times \mathbb{Z}_{n}\right)$, where $\mathbb{Z}_{n}$ is a finite cyclic group that permutes zeros of $A$.

Not surprisingly, coadjoint orbits of $H$ are more often discussed (mostly in the context of the Virasoro algebra $\hat{\mathfrak{h}}$ defined by central extension of $\mathfrak{h}$; see, for example, [Witten 88]). Elements of the regular dual space $\mathfrak{h}_{\text {reg }}^{*}$ of $\mathfrak{h}$ may be represented by quadratic differentials $p=$ $p(\theta) d \theta^{2}$ via the pairing

$$
\left(a \partial_{\theta}, p\right)=p(A)=\int_{S^{1}} a p d \theta
$$

When $p=w^{2} d \theta^{2}$ is nonvanishing along $S^{1}$, the kernel of

$$
B \mapsto \operatorname{ad}_{B}^{*} p=-\left(2 p b^{\prime}+p^{\prime} b\right) d \theta^{2}
$$

is spanned by $\frac{1}{w} \partial_{\theta} \in \mathfrak{h}$, and one has $\operatorname{Ad}_{H}^{*} p \simeq H / S^{1}$; thus, certain adjoint and coadjoint orbits resemble each other. (In the context of $\hat{\mathfrak{h}}$, the symplectic nature of $H / S^{1}$ leads to a well-known, remarkable derivation of the Korteweg-de Vries equation. Elsewhere, we will explore implications of such structure on the space of symmetric singular ring domains.)

For nonvanishing $A \in \mathfrak{h}$, we may define $\mathbf{p}_{A} \in \mathfrak{h}_{\text {reg }}^{*}$ by the formula

$$
\mathbf{p}_{A}(c)=\int_{0}^{2 \pi} \frac{c}{a^{2}} d \theta
$$

as the last paragraph suggests; but in general, the poles of $d \theta / a^{2}$ resulting from the zeros of $A$ interfere with the interpretation of this formula. Similarly, given $p \in \mathfrak{h}^{*}$ with a zero on $S^{1}, p$ is not stabilized by any continuous vector field and $\mathrm{Ad}_{H}^{*} p$ looks like (a finite quotient of) $H$ itself - unlike any adjoint orbit. The difference between adjoint and coadjoint actions has already been reflected above in the lack of a pseudo-Riemannian structure on $\Lambda$.

Remark 10.4. The kernel of $\operatorname{ad}_{A}: \mathfrak{g} \rightarrow \mathfrak{g}$ has a simple interpretation as the Lie algebra of the $S^{1}$-local isometry group of the foliated flat geometry defined by $p=\omega^{2}=d z^{2} /(f(z))^{2}$, where $A=f \partial_{z}$. (Such isometries are required to be defined only on a punctured neighborhood $A_{r} \backslash\left\{z_{1}, \ldots, z_{n}\right\}$ of $S^{1}$.) Namely, for any constant $\lambda \in \mathbb{C}^{\times}$, the vector field $\Re(\lambda W)$ generates a one-parameter local group $\left\{h_{t}\right\}$ satisfying $\operatorname{Ad}_{h_{t}} W=W$; hence $h_{t}^{*} \omega=\omega$, so $g=\omega \bar{\omega}$, and the horizontal foliation remain fixed.

If one relaxes the requirement that an isometry should preserve trajectories of $p=\omega^{2}$, that is, if one regards $\left(A_{r} \backslash\left\{z_{1}, \ldots, z_{n}\right\}, g=\omega \bar{\omega}\right)$ simply as a Riemannian manifold, then there are exceptional examples with threedimensional local isometry groups. To find such examples, first suppose a conformal map $h$ preserves the metric: $h^{*} g=g$, i.e., $\tilde{p}=h^{*} p=e^{2 i \theta} p$. Then the real function $\theta$ must be constant, since it is locally the ratio of holomorphic functions $\tilde{P}(z), P(z)$, and $\tilde{p}$ therefore belongs to the conjugate family $\left\{e^{2 i \theta} p\right\}, 0 \leq \theta<2 \pi$. If a holomorphic vector field $B=b \partial_{z}$ generates a one-parameter group $h_{t}$ of such maps, it induces a variation of the form $e^{i \theta(t)} \omega$. In this case $B$ satisfies $\operatorname{ad}_{B} W=i c W$, where $W=f \partial_{z}$ is the holomorphic vector field dual to $\omega=d z / f(z)$ and $c$ is a real constant.

In general, the formula $b=a \int c / a^{2}$ defines a solution $B=b \partial_{\theta} \in \mathfrak{h}$ to the equation $\operatorname{ad}_{B} A=C$ (uniquely up to multiples of $A$ ), provided the latter antiderivative yields a single-valued holomorphic function $b$ along $S^{1}$. (In the case of nonvanishing $A$, it follows that 
$\operatorname{im}\left(\operatorname{ad}_{A}\right)=\operatorname{ker}\left(\mathbf{p}_{A}\right)$, since $\mathbf{p}_{A}(c)=\int_{0}^{2 \pi} \frac{c}{a^{2}} d \theta=0$ is the condition for periodicity, $b(0)=b(2 \pi)$.) For the special equation $\operatorname{ad}_{B} W=i c W$, the formal solution

$$
b(z)=i c f(z) \int_{z_{0}}^{z} \frac{d \zeta}{f(\zeta)}
$$

yields a well-defined holomorphic vector field $B=b \partial_{z}$ near $S^{1}$ in the exceptional case that $\omega=d z / f(z)$ has vanishing $S^{1}$-period $\mathcal{I}=\Re\left[\int_{\gamma_{\epsilon}} \omega\right]$ and vanishing residues at all the poles $z_{1}, \ldots, z_{n} \in S^{1}$. In this case, $B$ is unique up to addition of terms $\lambda W$ already considered, and the combinations $\{c B+\lambda W\}$ generate a three-dimensional local isometry group. The simplest example of such exceptional behavior was already given in the last part of Example 4.1. More-complicated examples may easily be constructed as indicated, and it is amusing to picture Euclidean isometries associated with the various "infinities" $z_{j}$ coordinating to give an isometry on a neighborhood $A_{r} \backslash\left\{z_{1}, \ldots, z_{n}\right\}$.

\section{REFERENCES}

[Ablowitz and Fokas 97] M. Ablowitz and A. Fokas. Complex Variables, Cambridge Texts in Applied Mathematics. Cambridge: Cambridge University Press, 1997.

[Ahlfors 53] Lars Ahlfors. Complex Analysis, International Series in Pure and Applied Mathematics. New York: McGraw-Hill, 1953.

[Byrd and Friedman 54] Paul F. Byrd and Morris D. Friedman. Handbook of Elliptic Integrals for Engineers and Physicists. New York: Springer-Verlag, 1954.

[Calini and Langer 03] Annalisa Calini and Joel Langer. "Schwarz Reflection Geometry I: Continuous Iteration of Reflection." Math. Z. 244 (2003), 775-804.

[Cartan 63] Henri Cartan. Elementary Theory of Analytic Functions of One or Several Complex Variables. Reading, MA: Addison-Wesley.

[Davis 74] Philip J. Davis. The Schwarz Function and Its Applications, Carus Mathematical Monographs, 17. Washington, DC: Mathematical Association of America, 1974.

[Dieudonné 69] J. Dieudonné, Foundations of Modern Analysis, Pure and Applied Mathematics Series, 10 I. New York: Academic Press, 1969.
[Guest 97] Martin Guest. Harmonic Maps, Loop Groups, and Integrable Systems. London Mathematical Society Student Texts, 38. Cambridge: Cambridge University Press, 1997.

[Hirsch 76] Morris W. Hirsch. Differential Topology. Graduate Texts in Mathematics, 33, New York: Springer-Verlag, 1976.

[Kirillov 95] A. A. Kirillov. "Geometric Approach to Discrete Series of Unirreps for Vir." ESI preprint, 1995.

[Kobayashi and Wu 83] S. Kobayashi and H. Wu. Complex Differential Geometry. Cambridge: Birkhäuser, 1983.

[Krichever and Novikov 87] I. M. Krichever and S. P. Novikov. "Algebras of Virasoro Type, Riemann Surfaces and Structures of the Theory of Solitons." Functional Analysis and Its Applications 21:2 (1987), 126-142. Translation from Funktsional'nyi Analiz $i$ Ego Prilozheniya 21:2 (1987), 4663.

[Lehto and Virtanen 73] O. Lehto and K. I. Virtanen. Quasiconformal Mappings in the Plane. New York: SpringerVerlag, 1973.

[Loos 69] Ottmar Loos. Symmetric Spaces I: General Theory. New York: W. A. Benjamin, Inc., 1969.

[McMullen] C. McMullen. Complex Dynamics and Renormalization, Annals of Mathematics Studies, 135. Princeton, Princeton University Press, 1994.

[Milnor 84] John Milnor. "Remarks on Infinite Dimensional Lie Groups. In Relativity, Groups and Topology II, Les Houghes Session XL, 1983, edited by B. S. de Witt and R. Stora. Amsterdam: North-Holland, 1984.

[Moser 65] Jürgen Moser. "On the Volume Elements on a Manifold." TAMS 120:2 (1965), 286-294.

[Muciño-Raymundo 02] Jesus Muciño-Raymundo. "Complex Structures Adapted to Smooth Vector Fields." Math. Ann. 322 (2002), 229-265.

[Shapiro 92] Harold S. Shapiro. The Schwarz Function and Its Generalization to Higher Dimensions. University of Arkansas Lecture Notes in the Mathematical Sciences, 9. New York: John Wiley \& Sons, Inc., 1992.

[Sharpe 97] R. W. Sharpe. Differential Geometry. New York: Springer-Verlag, 1997.

[Springer 57] George Springer. Introduction to Riemann Surfaces. Reading MA: Addison-Wesley, 1957.

[Strebel 84] Kurt Strebel. Quadratic Differentials. New York: Springer-Verlag, 1984.

[Zygmund 59] A. Zygmund. Trigonometric Series, vol. II. Cambridge: Cambridge University Press, 1959.

[Witten 88] Edward Witten. "Coadjoint Orbits of the Virasoro Group." Comm. Math. Phys. 114 (1988), 1-53.

Annalisa Calini, Department of Mathematics, College of Charleston, Charleston, SC 29424 (calinia@cofc.edu)

Joel Langer, Department of Mathematics, Case Western Reserve University, Cleveland, OH 44106 (joel.langer@case.edu)

Received April 28, 2006; accepted October 18, 2006. 